\title{
Zero Actions and Energy Functions for Perfect Crystals
}

\author{
By
}

Timothy H. BAKER*

\begin{abstract}
We give a combinatorial description of the action of the crystal operators $\tilde{e_{0}}, \tilde{f}_{0}$ on certain perfect crystals of $U_{q}^{\prime}\left(C_{n}^{(1)}\right), U_{q}^{\prime}\left(D_{n}^{(1)}\right)$ and $U_{q}^{\prime}\left(D_{n+1}^{(2)}\right)$, by means of Dynkin diagram automorphisms and Schensted column insertion. Also, for certain level 1 perfect crystals of these algebras we give a definition of a combinatorial "charge" related to the energy function on homogeneous paths in such crystals.
\end{abstract}

\section{$\S 1 . \quad$ Introduction}

The theory of perfect crystals, initiated in [KMN1, KMN2] has provided the impetus for numerous investigations in various areas of science. Indeed, crystal base theory has turned up in areas as diverse as soliton cellular automata [HKT, FOY], and even the genetic code [FSS]! Its main application however, has been in the area of solvable lattice models. Rather than provide a comprehensive synopsis of role of crystal base theory in this area, we merely refer the reader to [HKOTY], where such a treatment is given, along with a large list of modern references.

For our purposes, it is enough to recall one of the defining characteristics of crystal bases, namely their nice behaviour under tensor products, given by the rule

Communicated by T. Miwa, December 7, 1999. Revised March 22, 2000.

1991 Mathematics Subject Classification(s): Primary 05E15, Secondary 81R50.

${ }^{*}$ Research Institute for Mathematical Sciences, Kyoto University, Kyoto 606-8502, Japan. 


$$
\begin{aligned}
& \tilde{f}_{\imath}\left(b_{1} \otimes b_{2}\right)= \begin{cases}\tilde{f}_{\imath}\left(b_{1}\right) \otimes b_{2} & \phi_{\imath}\left(b_{1}\right)>\varepsilon_{\imath}\left(b_{2}\right) \\
b_{1} \otimes \tilde{f}_{\imath}\left(b_{2}\right) & \phi_{\imath}\left(b_{1}\right) \leq \varepsilon_{\imath}\left(b_{2}\right)\end{cases} \\
& \tilde{e}_{\imath}\left(b_{1} \otimes b_{2}\right)= \begin{cases}\tilde{e}_{\imath}\left(b_{1}\right) \otimes b_{2} & \phi_{\imath}\left(b_{1}\right) \geq \varepsilon_{\imath}\left(b_{2}\right) \\
b_{1} \otimes \tilde{e}_{\imath}\left(b_{2}\right) & \phi_{\imath}\left(b_{1}\right)<\varepsilon_{\imath}\left(b_{2}\right)\end{cases}
\end{aligned}
$$

where $\phi_{\imath}(b)=\max \left\{k \mid\left(\tilde{f}_{\imath}\right)^{k} b \neq 0\right\}, \varepsilon_{\imath}(b)=\max \left\{k \mid\left(\tilde{e}_{\imath}\right)^{k} b \neq 0\right\}$. It follows from this rule, that

$$
\begin{aligned}
& \phi_{\imath}\left(b_{1} \otimes b_{2}\right)=\max \left(\phi_{\imath}\left(b_{2}\right), \phi_{\imath}\left(b_{2}\right)+\phi_{\imath}\left(b_{1}\right)-\varepsilon_{\imath}\left(b_{2}\right)\right) \\
& \varepsilon_{\imath}\left(b_{1} \otimes b_{2}\right)=\max \left(\varepsilon_{\imath}\left(b_{1}\right), \varepsilon_{\imath}\left(b_{1}\right)+\varepsilon_{\imath}\left(b_{2}\right)-\phi_{\imath}\left(b_{1}\right)\right)
\end{aligned}
$$

These formulae, along with the description of the crystal bases of irreducible representations of $U_{q}(\mathfrak{g})$, where $\mathfrak{g}$ is one of the classical Lie algebras, given by Kashiwara and Nakashima $[\mathrm{KN}]$ will be the main ingredients we shall require to present our results.

The purpose of this article is two-fold. In the first part, we consider the level $l$ perfect crystals of $U_{q}^{\prime}(\mathfrak{g})$ where $\mathfrak{g}$ is one of $C_{n}^{(1)}, D_{n}^{(1)}$ and $D_{n+1}^{(2)}$. Let $\mathfrak{g}_{0}$ be the classical part of $\mathfrak{g}$, so that $\mathfrak{g}_{0}$ is given by $C_{n}, D_{n}$ and $B_{n}$ respectively. Specifically, we consider the $U_{q}^{\prime}(\mathfrak{g})$ crystals which are isomorphic to $B\left(l \Lambda_{n}\right)$ (and $B\left(l \Lambda_{n-1}\right)$ in the case $\left.\mathfrak{g}_{0}=D_{n}\right)$ when considered as a $U_{q}\left(\mathfrak{g}_{0}\right)$ crystal. In reference $[\mathrm{KMN} 2]$, the zero action (action of the crystal operators $\tilde{e}_{0}$, $\tilde{f}_{0}$ ) on such crystals was defined in terms of a Dynkin diagram automorphism, say $\sigma$ (we use the same symbol to denote the action of the Dynkin automorphism on the roots of $\mathfrak{g}$ as well as on the crystal elements). The image of a crystal element $b$ under such an automorphism was computed by choosing a sequence of raising operators such that $b^{\prime}=\tilde{e}_{\imath_{1}} \cdots \tilde{e}_{i_{p}} b$ was a highest weight element for a certain subalgebra of $\mathfrak{g}_{0}$. The subalgebra was chosen such that the highest weight elements were multiplicity-free, in which case $\sigma\left(b^{\prime}\right)$ is uniquely determined from weight considerations alone. One can then reverse the above traversal so that $\sigma(b)=\tilde{f}_{\sigma\left(\imath_{p}\right)} \cdots \tilde{f}_{\sigma\left(\imath_{1}\right)} \sigma\left(b^{\prime}\right)$ can then be computed. Here, we shall give a more combinatorial approach using the Schensted (column) insertion procedure. Knowledge of the action of the Dynkin automorphism then allows one to compute the zero action since $\tilde{e}_{0} b=\sigma^{-1} \tilde{e}_{\sigma(0)} \sigma(b)$ and similarly for $\tilde{f}_{0}$. For the $U_{q}^{\prime}\left(C_{n}^{(1)}\right)$ and $U_{q}^{\prime}\left(D_{n+1}^{(2)}\right)$ crystals, we shall take advantage of the embeddings $C_{n}, B_{n} \hookrightarrow A_{2 n-1}$, while for the $U_{q}^{\prime}\left(D_{n}^{(1)}\right)$ crystals, we can compute the automorphism directly.

In the second part, we shall study the local energy function $H$ on certain level 1 crystals $B$ of the same types. In the case of the $U_{q}^{\prime}\left(A_{n}^{(1)}\right)$ perfect crystals ${ }^{1}$

\footnotetext{
${ }^{1}$ We use the notation of [HKOTY] with regards to the crystals $B^{r, s}$
} 
$B^{l, 1}$ and $B^{1, l}$ it was shown by Nakayashiki and Yamada [NY] how to associate to each classically restricted (in)homogeneous path (i.e. annihilated by $\tilde{e}_{1}$ for $1 \leq i \leq n) p=b_{1} \otimes \cdots \otimes b_{L}$ where each $b_{\imath} \in B$, a semi-standard tableau $T(p)$. Moreover, they were able to show that the Lascoux-Schützenberger charge [LS] of such a tableau was directly related to the energy of the corresponding path. This was subsequently generalized to the crystals $B^{k, l}$ by Schilling and Warnaar $[\mathrm{SW}]$ and Shimozono [Shi]. In this article, we consider the level 1 perfect crystals $B^{n, 1}$ of $U_{q}^{\prime}\left(C_{n}^{(1)}\right), U_{q}^{\prime}\left(D_{n}^{(1)}\right)$ and $U_{q}^{\prime}\left(D_{n+1}^{(2)}\right)$ and show how to associate to each finite, classically restricted, homogeneous path $p$ in $\left(B^{n .1}\right)^{\otimes L}$, a tableau $T(p)$. To such an object $T(p)$, we can define a charge such that charge $(T(p))$ is related to the energy $E(p)$ of the path $p$.

\section{§2. Dynkin Automorphisms}

\section{§2.1. $C_{n}^{(1)}$}

In reference [Kas], it was proven that certain $U_{q}(\mathfrak{g})$ crystals can be embedded into a $U_{q}(\mathfrak{h})$ crystal where $\mathfrak{g}$ is a subalgebra of $\mathfrak{h}$. In this section, we aim to describe explicitly the embedding of $U_{q}\left(C_{n}\right)$ crystals into $U_{q}\left(A_{2 n-1}\right)$ crystals. This in turn, will allow us to compute the action of the Dynkin automorphism on the $U_{q}^{\prime}\left(C_{n}^{(1)}\right)$ crystal $B^{n, l}$.

First, let us introduce some notation for ordered sets. By an ordered set, we mean a set $A:=\left\{a_{1}, \ldots, a_{p}\right\}$ such that $a_{1}<\cdots<a_{p}$. Let $|A|=p$ denote the cardinality of $A$, and let $A^{c}$ denote the complement of $A$ in the set $\{1, \ldots, n\}$. For an ordered set $A$, let $\bar{A}:=\left\{\overline{a_{p}}, \ldots, \overline{a_{1}}\right\}$. Given two ordered sets $A$ and $B$, define a partial order $A \leq B$ to mean that $|A| \geq|B|$ and that $a_{\imath} \leq b_{\imath}$ for $1 \leq i \leq|B|$ i.e. writing the entries from top to bottom, the two columns $A \mid B$ constitute a semi-standard tableaux in the usual sense.

Consider $A_{2 n-1}$ tableaux on the alphabet

$$
\mathcal{A}:=\{1, \ldots, n, \bar{n}, \ldots, \overline{1}\}, \quad 1<\cdots<n<\bar{n}<\cdots<\overline{1}
$$

and let $B_{A}\left(\lambda_{A}\right)$ be the $A_{2 n-1}$ crystal with highest weight

$$
\lambda_{A}=\sum_{\imath=1}^{n-1} m_{\imath}\left(\Lambda_{\imath}+\Lambda_{\overline{\imath+1}}\right)+2 m_{n} \Lambda_{n}
$$

If $\tilde{f}_{\imath}^{(A)}$, and $\tilde{f}_{\frac{j}{j}}^{(A)}$ denote the $A_{2 n-1}$ crystal operators which act on this crystal, then by defining

$$
S\left(\tilde{f}_{\imath}^{(C)}\right)=\left\{\begin{array}{cc}
\tilde{f}_{\imath}^{(A)} \tilde{f}_{\imath+1}^{(A)} & 1 \leq i<n \\
\left(\tilde{f}_{n}^{(A)}\right)^{2} & i=n
\end{array}\right.
$$




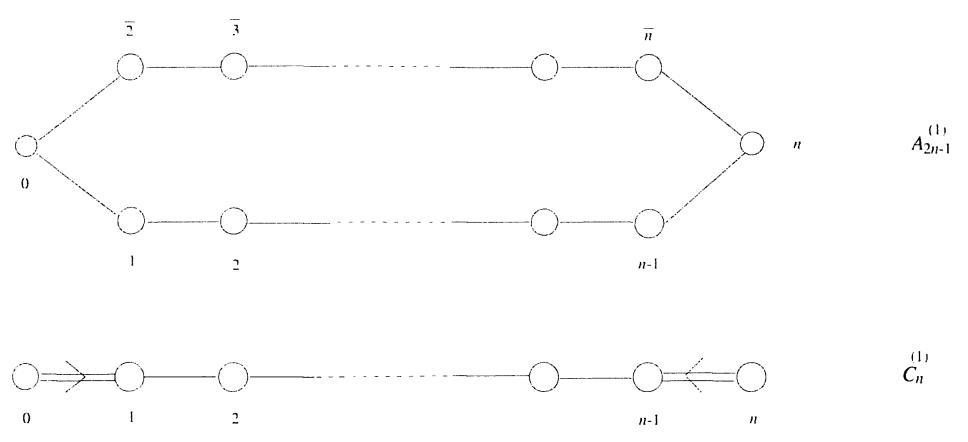

Figure 2.1. Dynkin diagrams for $A_{2 n-1}^{(1)}$ and $C_{n}^{(1)}$.

then the crystal generated by acting with the lowering operators $S\left(\tilde{f}_{i}^{(C)}\right)$ on the highest weight element $u_{\lambda_{A}}$ of $B_{A}\left(\lambda_{A}\right)$, is isomorphic to the $U_{q}\left(C_{n}\right)$ crystal $B_{C}\left(\lambda_{C}\right)$ with highest weight $\lambda_{C}=\sum_{i} m_{i} \Lambda_{i}$. In other words, we have an embedding $E: B_{C}\left(\lambda_{C}\right) \hookrightarrow B_{A}\left(\lambda_{A}\right)$. The explicit description of the $U_{q}\left(A_{2 n-1}\right)$ and $U_{q}\left(C_{n}\right)$ crystals we shall be using here, along with the action of the crystal operators $\tilde{e}_{i}, \tilde{f}_{i}$ can be found in $[\mathrm{KN}]$.

To describe this embedding explicitly, let us first begin with the one-column case, so that $\lambda_{C}=\Lambda_{k}$ for some fixed $k$, with $1 \leq k \leq n$, and hence $\lambda_{A}=$ $\Lambda_{k}+\Lambda_{\overline{k+1}}$ for $1 \leq k<n$ and $\lambda_{A}=2 \Lambda_{n}$ for $k=n$. Thus, the elements in the $U_{q}\left(C_{n}\right)$ crystal $B_{C}\left(\lambda_{C}\right)$ are given by tableaux consisting of one column of length $k$, satisfying the conditions specified in $[\mathrm{KN}]$, while the elements in the $U_{q}\left(A_{2 n-1}\right)$ crystal $B_{A}\left(\lambda_{A}\right)$ are given by the usual 2-column semi-standard tableaux with column lengths $2 n-k$ and $k$.

Given a $C_{n}$ column $P$ say, let $P_{+}$(resp. $\overline{P_{-}}$) denote the set of unbarred (resp. barred) elements appearing in $b$. Following [She], we define two new sets $Q_{ \pm}$as follows. Let

$$
K:=P_{+} \cap P_{-}, J:=\max \left\{A \subseteq\left(P_{+} \cup P_{-}\right)^{c}|\quad| A|=| K \mid \text { and } A<K\right\}
$$

Here, the maximum is taken with respect to the partial order on ordered sets, and $P^{c}:=\{1, \ldots, n\} / P$. Note that $J$ is well-defined, since the fact that at least one such set $A$ exists is equivalent to the fact that the set $P$ obeys the one-column condition (1CC) for $C_{n}$ tableaux (see (2.9)). Having calculated $J$ and $K$, set

$$
Q_{ \pm}:=\left(P_{ \pm} / K\right) \cup J
$$

The construction of the sets $Q_{ \pm}$can be visualized in the following way: con- 
sider two parallel vertical lines with the entry $i \in P_{+}$(resp. $P_{-}$) denoted by "particles" at position $i$ on the left (resp. right) line, and all other positions are considered "holes". Starting with the highest pair of particles on the same level, move the particles downwards into the highest "pair" of holes. Repeat this for the remaining pairs of particles from top to bottom. The left (resp. right) vertical line now gives the entries in $Q_{+}$(resp. $Q_{-}$).

Note the following useful properties

Lemma 2.1. We have

1. If $P:=\left\{i_{1}, \ldots, i_{p}, \overline{j_{q}}, \ldots, \bar{j}_{1}\right\} \in B_{C}\left(\Lambda_{k}\right)$ then for any $m, l$, we have

$$
m+l \leq \max \left(i_{m}, j_{l}\right)
$$

2. If $Q:=\left\{i_{1}, \ldots, i_{p}, \bar{j}_{q}, \ldots, \bar{j}_{1}\right\}$ is defined as above, then for any $m, l$, we have

$$
m+l \geq\left(\min \left(i_{m}, j_{l}\right)-1+(p+q)-n\right)_{+}+2
$$

where $(x)_{+}=\max (x, 0)$.

Proof. 1 is given in [KN, Lemma 4.3.1], while 2 follows from the definition of $Q$, using a similar argument.

Using this Lemma, we can now prove the following

Proposition 2.2. Define a map $\psi$ by

$$
\psi(P):=\begin{array}{l|l}
P_{-}^{c} & P_{+} \\
\left(\bar{Q}_{+}\right) & c \\
\bar{Q}_{-}
\end{array}
$$

Then $\psi: B_{C}\left(\Lambda_{k}\right) \hookrightarrow B_{A}\left(\Lambda_{k}+\Lambda_{2 n-k}\right)$ is a crystal embedding.

Proof. We first show that $\psi(P)$ is a well defined $A_{2 n-1}$ tableau. Certainly the strictly increasing vertical condition is satisfied by definition. For the weakly increasing horizontal condition, note that $\left|P_{-}^{c}\right|=\left|P_{+}\right|+n-k \geq\left|P_{+}\right|$. We shall first show that the subtableau $P_{-}^{c} \mid P_{+}$satisfies the horizontal condition. Let $P_{+}=\left\{i_{1}, \ldots, i_{p}\right\}$. Given $i_{m} \in P_{+}$, we shall show that the $m$ 'th entry of $P_{-}^{c}$, call it $\left(P_{-}^{c}\right)_{m}$ say, satisfies $\left(P_{-}^{c}\right)_{m} \leq i_{m}$. Let $j_{l}$ be the largest integer $\leq m$ appearing in $P_{-}$, if such an integer exists (if no such $j_{l}$ exists, then $\{1, \ldots, m\} \subseteq P_{-}^{c}$ and so $\left(P_{-}^{c}\right)_{m}=m \leq i_{m}$ and we are done). From Lemma 2.1 we have

$$
m+l \leq \max \left(i_{m}, j_{l}\right)=i_{m}
$$


Hence we have

$$
\left(P_{-}^{c}\right)_{m}=m+\#\left\{j \in P_{-} \mid j \leq m\right\}=m+l \leq i_{m}
$$

Let us now show that the tableau $\left(\bar{Q}_{+}\right)^{c} \mid \bar{Q}_{-}$satisfies the horizontal condition (the result will then follow as in the tableau (2.3), the column $\left(\bar{Q}_{+}\right)^{c}$ is shifted down by $n-k$ positions, which preserves the horizontal condition). Let $\bar{Q}_{-}=\left\{\bar{j}_{q}, \ldots, \bar{j}_{1}\right\}$. Given $\bar{j}_{l}$, let $\bar{x}$ be the entry immediately to its left in the column $\left(\bar{Q}_{+}\right)^{c}$. That is, $\bar{x}$ is the $(q-l)$ 'th entry of $\left(\bar{Q}_{-}\right)^{c}$. We must show $\bar{x} \leq \bar{j}_{l}$ or equivalently $x \geq j_{l}$. Let $i_{m}$ be the smallest integer $\geq n-(q-l)$ which appears in $Q_{+}$(again, if no such $i_{m}$ exists, then $\{n-q+l, \ldots, n\} \subseteq Q_{+}^{c}$ and so $x=n-q+l \geq j_{l}$ and we are done). Now Lemma 2.1 gives

$$
m+l \geq\left(j_{l}-1+p+q-n\right)_{+}+2
$$

Thus

$$
\begin{aligned}
x & =n-q+l-\#\left\{i \in Q_{+} \mid i \geq n-q-l\right\} \\
& =n-q+l-(p+1-m) \geq j_{l}
\end{aligned}
$$

hence the result.

It remains to show that

$$
\psi\left(\tilde{f}_{\imath}^{(C)} P\right)=S\left(\tilde{f}_{l}^{(C)}\right) \psi(P)
$$

where $S\left(\tilde{f}_{\imath}^{(C)}\right)$ is defined in (2.2). For $i$ such that $1 \leq i \leq n-1$, one must consider the 16 possible cases corresponding to the presence or absence of $i$, $i+1$ in $P_{+}$and $P_{-}$. Similarly there are 4 cases to consider when $i=n$. As a representative example, let us choose the case where $1 \leq i \leq n-1$ and

$$
P=\{\ldots, i, i+1, \ldots, \overline{i+1}, \ldots\}
$$

in which case, we have

$$
Q=\{\ldots, \stackrel{k}{i}, \ldots\}
$$

where $i$ is in the $k^{\prime}$ 'th position in $Q$. Apply $\tilde{f}_{\imath}^{(C)}$ we have

$$
P^{\prime}=\tilde{f}_{\imath}^{(C)} P=\{\ldots, i, i+1, \ldots, \bar{i}, \ldots\}
$$

and so

$$
Q^{\prime}=\{\ldots, i \stackrel{k}{+} 1, \ldots\}
$$


The point is that, from the definition of $Q$, the entry $i+1$ in $Q^{\prime}$ is also in the $k$ 'th position, and all other entries remain unchanged. Thus, we have

$$
\psi(P)=\begin{array}{c|cc|c}
i & i & i+1 & i \\
\vdots & i+1 & \psi\left(P^{\prime}\right)= & i+1 \\
\hline i+1 & \vdots & \bar{i} & \vdots
\end{array}
$$

and all other entries not displayed are the same in both $\psi(P)$ and $\psi\left(P^{\prime}\right)$. Thus (2.4) is indeed satisfied. The other cases are proved in a similar manner.

Remark. In the course of verifying each of the separate cases in the above proof, it can be checked that we have the following relations

$$
\phi_{\imath}^{(C)}(b)=\left\{\begin{array}{cc}
\phi_{\imath}^{(A)}(\psi(b))=\phi \frac{(A)}{1+1}(\psi(b)) & 1 \leq i<n \\
\frac{1}{2} \phi_{n}^{(A)}(\psi(b)) & i=n
\end{array}\right.
$$

and similarly for $\varepsilon_{\imath}^{(C)}(b)$.

Let us now turn to the general case. Recall the notion of a tableaux product [Ful]. Given two semi-standard (i.e. type A) tableaux $S, T$, define the tableaux product

$$
S * T=\left(\cdots\left(\left(S \leftarrow a_{1}\right) \leftarrow a_{2}\right) \cdots\right) \leftarrow a_{p}
$$

where $w(T)=a_{1} a_{2} \cdots a_{p}$ is the Japanese/Chinese reading of $T$ (i.e. reading the columns from top to bottom, and right to left). It is well-known that $*$ induces an isomorphism of $U_{q}\left(A_{n-1}\right)$ crystals. Suppose we are given an element $b \in B_{C}(\lambda)$ where $\lambda=\Lambda_{m_{1}}+\cdots+\Lambda_{m_{p}}$, where $m_{1} \leq \cdots \leq m_{p}$. Then we can write $b=b_{1} \otimes \cdots \otimes b_{p}$, where $b_{\imath} \in B_{C}\left(\Lambda_{m_{1}}\right)$ is the $i$ 'th column of the tableau $b$ reading from right to left.

Proposition 2.3. Define a map $E$ on $B_{C}(\lambda)$ by

$$
E(b):=\psi\left(b_{1}\right) * \psi\left(b_{2}\right) * \cdots * \psi\left(b_{p}\right)
$$

where $\psi$ is defined in (2.3). Then we have

(i) $E: B_{C}(\lambda) \hookrightarrow B_{A}\left(\lambda^{\prime}\right)$ where $\lambda^{\prime}=\sum_{\imath=1}^{p}\left(\Lambda_{m},+\Lambda_{2 n-m_{1}}\right)$ is a crystal embedding. 
(ii)

$$
\phi_{\imath}^{(C)}(b)=\left\{\begin{array}{cc}
\phi_{\imath}^{(A)}(E(b))=\phi \frac{(A)}{\imath+1}(E(b)) & 1 \leq i<n \\
\frac{1}{2} \phi_{n}^{(A)}(E(b)) & i=n
\end{array}\right.
$$

and similarly for $\varepsilon_{\imath}^{(C)}(b)$.

Proof. In order to prove (i), we must show

$\left(\mathrm{i}^{\prime}\right) E\left(\tilde{e}_{\imath} b\right)=S\left(\tilde{e}_{2}\right) E(b)$, for $1 \leq i \leq n$.

$\left(\mathrm{i}^{\prime \prime}\right)$ If $E(b)$ is annihilated by all $S\left(\tilde{e}_{\imath}\right)$, then $E(b)$ is the (unique) highest weight element of $B_{A}\left(\lambda^{\prime}\right)$.

We prove $\left(\mathrm{i}^{\prime}\right),\left(\mathrm{i}^{\prime \prime}\right)$ and (ii) simultaneously by induction on $p$, noting that the case $p=1$ follows from Prop. 2.2 and (2.5). Write $b=b^{\prime} \otimes b_{p}$ where $b^{\prime}=$ $b_{1} \otimes \cdots \otimes b_{p-1}$. Note that for $1 \leq i \leq n-1$, we have from (1.2), (2.5) and the induction hypothesis

$$
\phi_{\imath}^{(C)}\left(b^{\prime} \otimes b_{p}\right)=\phi_{\imath}^{(A)}\left(E\left(b^{\prime}\right) \otimes \psi\left(b_{p}\right)\right)=\phi_{\imath}^{(A)}\left(E\left(b^{\prime} \otimes b_{p}\right)\right)
$$

where the last equality follows from the fact that $*$ is a crystal isomorphism. A similar argument holds for the case $i=n$ (since the function max is linear with respect to scalar multiplication), as well as for the cases for $\varepsilon_{\imath}^{(C)}(b)$. Hence (ii) holds. To show $\left(\mathrm{i}^{\prime}\right)$, we use the tensor product rule (1.1) twice and note from the induction hypothesis that $\phi_{\imath}^{(A)}\left(E\left(b^{\prime}\right)\right)=\phi \frac{(A)}{\imath+1}\left(E\left(b^{\prime}\right)\right)$ etc for $1 \leq i<n$, so that

$$
\begin{aligned}
S\left(\tilde{e}_{\imath}\right)\left(E\left(b^{\prime}\right) \otimes \psi\left(b_{p}\right)\right) & = \begin{cases}S\left(\tilde{e}_{\imath}\right) E\left(b^{\prime}\right) \otimes \psi\left(b_{p}\right) & \phi_{\imath}^{(A)}\left(E\left(b^{\prime}\right)\right) \geq \varepsilon_{\imath}^{(A)}\left(\psi\left(b_{p}\right)\right) \\
E\left(b^{\prime}\right) \otimes S\left(\tilde{e}_{\imath}\right) \psi\left(b_{p}\right) & \phi_{\imath}^{(A)}\left(E\left(b^{\prime}\right)\right)<\varepsilon_{\imath}^{(A)}\left(\psi\left(b_{p}\right)\right)\end{cases} \\
& = \begin{cases}E\left(\tilde{e}_{\imath} b^{\prime} \otimes b_{p}\right) & \phi_{\imath}^{(C)}\left(b^{\prime}\right) \geq \varepsilon_{\imath}^{(C)}\left(b_{p}\right) \\
E\left(b^{\prime} \otimes \tilde{e}_{\imath} b_{p}\right) & \phi_{\imath}^{(C)}\left(b^{\prime}\right)<\varepsilon_{\imath}^{(C)}\left(b_{p}\right)\end{cases} \\
& =E\left(\tilde{e}_{\imath}\left(b^{\prime} \otimes b_{p}\right)\right)
\end{aligned}
$$

where the second line also follows from induction. The case $i=n$ follows similarly. Finally, to show $\left(i^{\prime \prime}\right)$, recall [Nak, Prop. 3.2.1] that

$$
\tilde{e}_{\imath}(u \otimes v)=0, \forall i \quad \Leftrightarrow \quad \tilde{e}_{\imath}(u)=0, \text { and } \varepsilon_{\imath}(v) \leq\left\langle h_{\imath}, w t(u)\right\rangle \quad \forall i
$$

Thus, if $S\left(\tilde{e}_{\imath}\right) E\left(b^{\prime} \otimes b_{p}\right)=0 \forall i$, then $S\left(\tilde{e}_{\imath}\right)\left(E\left(b^{\prime}\right) \otimes \psi\left(b_{p}\right)\right)=0 \forall i$, and hence (a) $S\left(\tilde{e}_{\imath}\right) E\left(b^{\prime}\right)=0 \Rightarrow \tilde{e}_{\imath} b^{\prime}=0$ by $\left(\mathrm{i}^{\prime}\right)$ and the fact that $E$ is injective, (b) 
$\varepsilon_{\imath}^{(A)}\left(\psi\left(b_{p}\right)\right) \leq\left\langle h_{\imath}^{(A)}, w t\left(E\left(b^{\prime}\right)\right)\right\rangle \Rightarrow \varepsilon_{\imath}^{(C)}\left(b_{p}\right) \leq\left\langle h_{\imath}^{(C)}, w t\left(b^{\prime}\right)\right\rangle$ by (ii) and the fact that $\left\langle h_{\imath}^{(A)}, w t\left(E\left(b^{\prime}\right)\right)\right\rangle=\left\langle h_{\imath}^{(C)}, w t\left(b^{\prime}\right)\right\rangle=m_{\imath}$. Hence by $(2.7)$ again we have that $b^{\prime} \otimes b_{p}$ is a highest weight element of the $C_{n}$ crystal $B_{C}(\lambda), \lambda=\sum_{\imath=1}^{p} \Lambda_{m}$, (i.e. the tableau with the entries $1, \ldots, m_{\imath}$ in the $i$ 'th column reading from right to left). Explicitly computing $E$ on this tableau then gives the required result.

Example. Let $n=6$, and consider the $C_{6}$ tableau

$$
T=\begin{array}{|l|l|l|}
\hline 2 & 2 & 4 \\
\hline 4 & 4 & \overline{6} \\
\hline 5 & \overline{6} & \overline{5} \\
\hline 6 & \overline{5} & \overline{4} \\
\hline \overline{5} & \overline{1} & \multicolumn{1}{|l}{} \\
\cline { 1 - 2 } \overline{3} & \multicolumn{2}{|l}{} \\
\cline { 1 - 1 }
\end{array}
$$

We first compute the image of each column under $\psi$. For the first column,

$$
P=\{2,4,5,6, \overline{5}, \overline{3}\}, \quad Q=\{1,2,4,6, \overline{3}, \overline{1}\}
$$

and hence

$$
\psi\left(\begin{array}{l|}
\hline 2 \\
\hline 4 \\
\hline 5 \\
\hline 6 \\
\hline \overline{5} \\
\hline \overline{3}
\end{array}\right)=\begin{array}{|l|l|}
\hline 1 & 2 \\
\hline 2 & 4 \\
\hline 4 & 5 \\
\hline 6 & 6 \\
\hline \overline{5} & \overline{3} \\
\hline \overline{3} & \overline{1} \\
\hline
\end{array}
$$

The action of $\psi$ on the second and third columns can be computed similarly. Combining these, we have

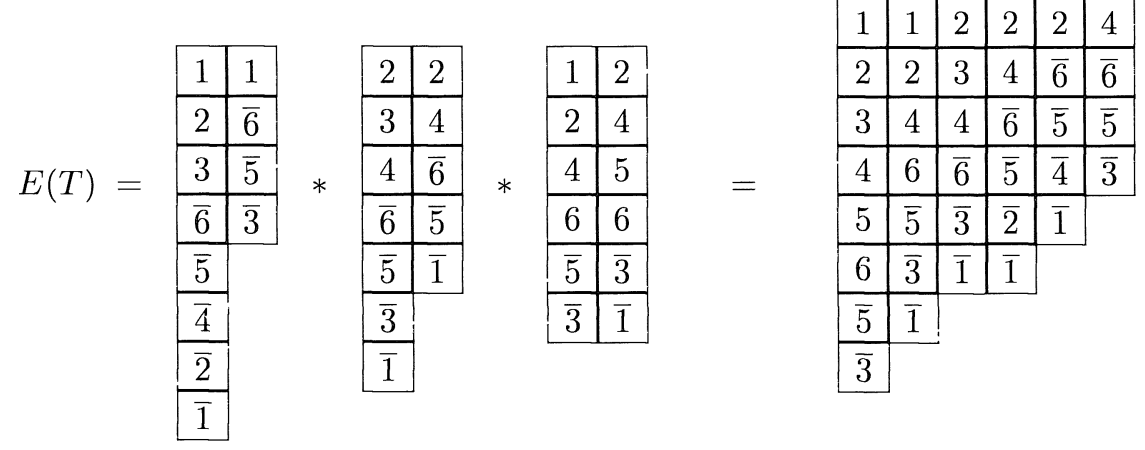


Turning to the perfect case, i.e. the crystal $B_{C}\left(l \Lambda_{n}\right)$, we shall see there are some simplifications. First note that in this case, we have

$$
\left(P_{-}\right)^{c}=Q_{+}
$$

To prove this, define the sets $K:=P_{+} \cap P_{-}, H:=P_{+}^{c} \cap P_{-}^{c}$ and $S:=\left(P_{+} / P_{-}\right) \cup$ $\left(P_{-} / P_{+}\right)$. Thus $K, H$ and $S$ denote the positions of the pairs of "particles", "holes" and the "singlets" in the description of $P_{ \pm}$given earlier. Certainly we must have $|K|+|H|+|S|=n$. Also, in the case of single column tableaux of length $n$, we must also have $2|K|+|S|=n$. That is, $|K|=|H|$. For a set $A$, let $A_{\leq x}:=\{y \in A \mid y \leq x\}$, and similarly for $A_{\geq x}$. The 1CC for $C_{n}$ tableaux is equivalent to the statement that

$$
\left|H_{\leq x}\right| \geq\left|K_{\leq x}\right| \quad \forall x \in\{1, \ldots, n\}
$$

Returning to the proof of (2.8), first note that we have $Q_{+} \subseteq P_{-}^{c}$ (this is true in the non-perfect case also) since if $x \in Q_{+}$, then either $x \in P_{+} \backslash P_{-}$, or $x \in$ $P_{+}^{c} \cap P_{-}^{c}$, and hence in both cases $x \in P_{-}^{c}$. Conversely, let us assume $P_{-}^{c} \nsubseteq Q_{+}$ and find a contradiction. In such a situation, there exists $y \in H$ such that after constructing $Q_{ \pm}$, the holes at level $y$ remain unoccupied. In particular, this means that $\left|K_{\geq y+1}\right| \leq\left|H_{\geq y+1}\right|$. Thus, since $\left|K_{\geq y+1}\right|+\left|K_{\leq y-1}\right|=|K|=|H|=$ $1+\left|H_{\geq y+1}\right|+\left|H_{\leq y-1}\right|$, we have $\left|K_{\leq y-1}\right| \geq 1+\left|H_{\leq y-1}\right|$, which contradicts (2.9). Hence (2.8) does indeed hold.

Recall [Con, She] that the $C_{n}$ tableaux of Kashiwara and Nakashima have another description in terms of certain "double" tableaux of De Concini. More precisely, it was shown in [She] that a $C_{n}$ tableau of $\mathrm{K}-\mathrm{N}$ has column form $P^{(1)}\left|P^{(2)}\right| \cdots \mid P^{(l)}$ if and only if the tableau

$$
\begin{array}{l|l|l|l|l|l|l}
Q_{+}^{(1)} & P_{+}^{(1)} & Q_{+}^{(2)} & P_{+}^{(2)} & \cdots & Q_{+}^{(l)} & P_{+}^{(l)} \\
\bar{P}_{-}^{(1)} & \bar{Q}_{-}^{(1)} & \bar{P}_{-}^{(2)} & \bar{Q}_{-}^{(2)} & \cdots & \bar{P}_{-}^{(l)} & \bar{Q}_{-}^{(l)}
\end{array}
$$

is a valid $A_{2 n-1}$ tableau. It follows from the definition of Schensted column insertion, that in the perfect case when (2.8) holds, the image of $b \in B_{C}\left(l \Lambda_{n}\right)$ under $E$ is precisely (2.10).

Having described the embedding $E$ for $U_{q}\left(C_{n}\right)$ crystals into $U_{q}\left(A_{2 n-1}\right)$ crystals, we can now use this to compute the $C_{n}^{(1)}$ Dynkin automorphism and hence the zero action on perfect $U_{q}^{\prime}\left(C_{n}^{(1)}\right)$ crystals $B^{n, l}$. From the correspondence $S$ (see (2.2)) between the Dynkin diagrams of $A_{2 n-1}^{(1)}$ and $C_{n}^{(1)}$, we have that $\sigma_{C}=\sigma_{A}^{n}$. Now, it is known [Shi] that for rectangular type $A$ tableaux, the automorphism $\sigma_{A}$ is given by the promotion operator $p r$ defined on an $A_{N}$ tableau $T$ by the following procedure 
1. replace all entries $N$ in $T$ by empty boxes and reverse slide them into the north-west corner of the tableau using jeu-de-taquin.

2. Place the entry 1 in these empty boxes and add one to all other entries in the tableau (for the alphabet $\{1, \ldots, n, \bar{n}, \ldots, 1\}$ this means $i \mapsto i+1$, $\overline{i+1} \mapsto \bar{i}$ for $1 \leq i \leq n-1$ and $n \mapsto \bar{n})$.

Thus we have $\sigma_{C}=E^{-1}(p r)^{n} E$ acting on the crystals $B^{n, l}$. Moreover, to compute the action of $\tilde{f}_{0}^{(C)}$ on this crystal, we have

$$
\begin{aligned}
S\left(\tilde{f}_{0}\right) & =\sigma_{C}^{-1} S\left(\tilde{f}_{n}\right) \sigma_{C}=E^{-1}(p r)^{-n}\left(\tilde{f}_{n}^{(A)}\right)^{2}(p r)^{n} E \\
& =E^{-1} p^{-1}\left(\tilde{f}_{1}^{(A)}\right)^{2} \operatorname{pr} E
\end{aligned}
$$

\section{§2.2. $D_{n}^{(1)}$}

In this subsection, we consider the perfect crystals $B^{n, l}$ (resp. $B^{n-1, l}$ ) whose non-affine part are the $U_{q}\left(D_{n}\right)$ crystals $B\left(l \Lambda_{n}\right)$, (resp. $\left.B\left(l \Lambda_{n-1}\right)\right)$. First recall $[\mathrm{KN}]$ the crystals of the fundamental spin representations of $U_{q}\left(D_{n}\right)$. The elements of the crystal $B\left(\Lambda_{n}\right)$ (resp. $B\left(\Lambda_{n-1}\right)$ ) can be represented by a single column tableaux $b$ of length $n$ on the alphabet (2.1), subject to the condition that the entries are strictly increasing down the column, and that the entries $i$ and $\bar{i}$ do not appear simultaneously for each $i, 1 \leq i \leq n$. Also, let the column reading of $b$ be $\left(\alpha_{1}, \ldots, \alpha_{n}\right)$. Then if the entry $\alpha_{k}=n$ then $n-k$ is even (resp. odd) and if $\alpha_{k}=\bar{n}$ then $n-k$ is odd (resp. even). Note that since the column length is $n$, the set of barred and unbarred elements are complements in the set $\{1, \ldots, n\}$.

For the level $l$ crystals $B\left(l \Lambda_{n}\right), B\left(l \Lambda_{n-1}\right)$, we follow [KMN2] and consider such crystals as being embedded inside $B\left(\Lambda_{n}\right)^{\otimes l}\left(\right.$ resp. $\left.B\left(\Lambda_{n-1}\right)^{\otimes l}\right)$. As such, we have the following description

Proposition 2.4. The crystal $B\left(l \Lambda_{n}\right)$ (resp. $B\left(l \Lambda_{n-1}\right)$ ) is isomorphic to the set of semi-standard tableaux on the alphabet $\{1, \ldots, n\}$ whose shape is contained in the rectangle $\left(l^{n}\right)$, and whose column lengths are even/odd (resp. odd/even) if $n$ is even/odd, with the following action of $\tilde{e}_{\imath}, \tilde{f}_{\imath}$

1. for $1 \leq i \leq n-1$, the action of $\tilde{e}_{2}, \tilde{f}_{2}$ is the same as for the $A_{n-1}$ case

2. if $i=n$, then reading the columns from right to left, assign an opening (resp. closing) parenthesis "(" (resp. ")") if the column contains both (resp. neither) $n-1$ and $n$. After computing the reduced word by recursively eliminating matching brackets "()", $\tilde{e}_{n}$ (resp. $\tilde{f}_{n}$ ) acts on the column 


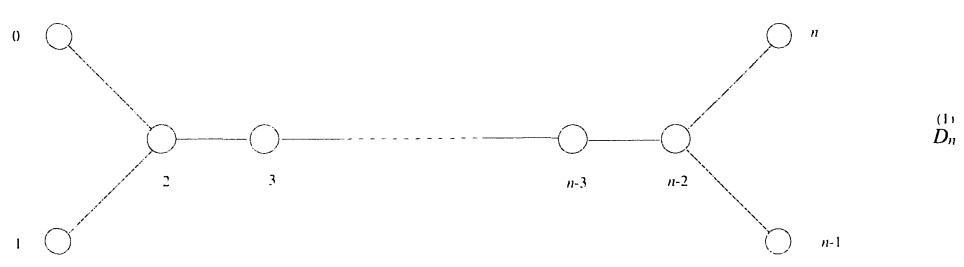

Figure 2.2. Dynkin diagram for $D_{n}^{(1)}$.

corresponding to the rightmost ")" (resp. leftmost "(") by appending (resp. deleting) the entries $n-1$ and $n$.

Proof. If $b$ is an $A_{n-1}$ tableau with even/odd column lengths for $n$ even/odd, whose shape is contained in the rectangle $\left(l^{n}\right)$, then we can associate a unique element $b_{1} \otimes \cdots \otimes b_{l} \in B\left(\Lambda_{n}\right)^{\otimes l}$ such that unbarred entries of $b_{i}$ are given by the $i$ 'th column (reading from right to left) of $b$. That such elements $b$ are closed under the action of $\tilde{e}_{i}, \tilde{f}_{i}$ described above and are connected by raising operators to the highest weight element of $B\left(l \Lambda_{n}\right)$ follows by similar arguments as given in $[\mathrm{KN}]$. The case of $B\left(l \Lambda_{n-1}\right)$ is similar.

Note that in the above description of $B\left(l \Lambda_{n}\right)$, the element of $B\left(\Lambda_{n}\right)$ with reading $(\bar{n}, \ldots, \overline{2}, \overline{1})$ corresponds to an empty column, so if the number of columns $p$ of $b$ is less than $l$, then the last $l-p$ "empty" columns correspond to such lowest weight elements of $B\left(\Lambda_{n}\right)$ (such a situation doesn't arise for $B\left(l \Lambda_{n-1}\right)$ since in that case, the lowest weight element of $B\left(\Lambda_{n-1}\right)$ has reading $\left.(n, \overline{n-1}, \ldots, \overline{1})\right)$.

Let us now consider the automorphism of the Dynkin diagram for $D_{n}^{(1)}$ which sends $\alpha_{i} \mapsto \alpha_{n-i}$. Using the above description of the crystals $B\left(l \Lambda_{n-1}\right)$ and $B\left(l \Lambda_{n}\right)$ we can compute the image of such an automorphism using Schensted insertion.

Proposition 2.5. If $b_{j} \in B\left(\Lambda_{n}\right)$ or $B\left(\Lambda_{n-1}\right)$, let $I=\left(i_{1}, i_{2}, \ldots, i_{p}\right)$ be its column reading (of the unbarred entries) and let $I^{c}=\left(i_{1}^{\prime}, i_{2}^{\prime}, \ldots, i_{q}^{\prime}\right)$ be its complement in $\{1, \ldots n\}$. Define a map $\omega$ by

$$
\omega\left(i_{1}, i_{2}, \ldots, i_{p}\right)=\left(n+1-i_{q}^{\prime}, \ldots, n+1-i_{2}^{\prime}, n+1-i_{1}^{\prime}\right)
$$

For $b \in B\left(l \Lambda_{n}\right)$ or $B\left(l \Lambda_{n-1}\right)$ write $b=b_{1} \otimes \cdots \otimes b_{l}$ and define

$$
\sigma_{D}(b)=\omega\left(b_{1}\right) * \cdots * \omega\left(b_{l}\right)
$$


where $*$ denotes the $A_{n-1}$ tableaux product defined in (2.6). Then $\sigma_{D}$ satisfies $\sigma_{D} \tilde{f}_{\imath}=\tilde{f}_{n-\imath} \sigma_{D}$ for $1 \leq i \leq n-1$, and similarly for $\tilde{e}_{\imath}$. Moreover, $\sigma_{D}^{2}=1$.

Proof. We proceed by induction on $l$. For $l=1$, fix an integer $i$ such that $1 \leq i<n$. Then by considering the 4 cases corresponding to whether $b_{1}$ contains $i$ and/or $i+1$, the result follows directly from the definition.

Now assume the result holds for perfect crystals of level $<l$. Then for such crystals, we certainly have

$$
\phi_{\imath}\left(\sigma_{D}(b)\right)=\phi_{n-\imath}(b), \quad \varepsilon_{\imath}\left(\sigma_{D}(b)\right)=\varepsilon_{n-\imath}(b), \quad 1 \leq i<n
$$

By using the tensor product rule (1.1) along with (2.11) the fact that $*$ is a $A_{n-1}$ crystal isomorphism and Prop. 2.4, the result follows.

Example. Let $n=8$, and consider the following element in $B\left(4 \Lambda_{8}\right)$

$$
\begin{aligned}
b= & (\overline{8}, \overline{7}, \overline{6}, \overline{5}, \overline{4}, \overline{3}, \overline{2}, \overline{1}) \otimes(3,6,7,8, \overline{5}, \overline{4}, \overline{2}, \overline{1}) \\
& \otimes(2,4,5,7, \overline{8}, \overline{6}, \overline{3}, \overline{1}) \otimes(1,3,4,5,6,8, \overline{7}, \overline{2})
\end{aligned}
$$

This corresponds to the $A_{7}$ tableau

$$
b=\begin{array}{|l|l|l|}
\hline 1 & 2 & 3 \\
\hline 3 & 4 & 6 \\
\hline 4 & 5 & 7 \\
\hline 5 & 7 & 8 \\
\hline 6 & \multicolumn{2}{|l}{} \\
\cline { 1 - 1 } 8 & \multicolumn{2}{|l}{} \\
\cline { 1 - 1 }
\end{array}
$$

By the above proposition, we have

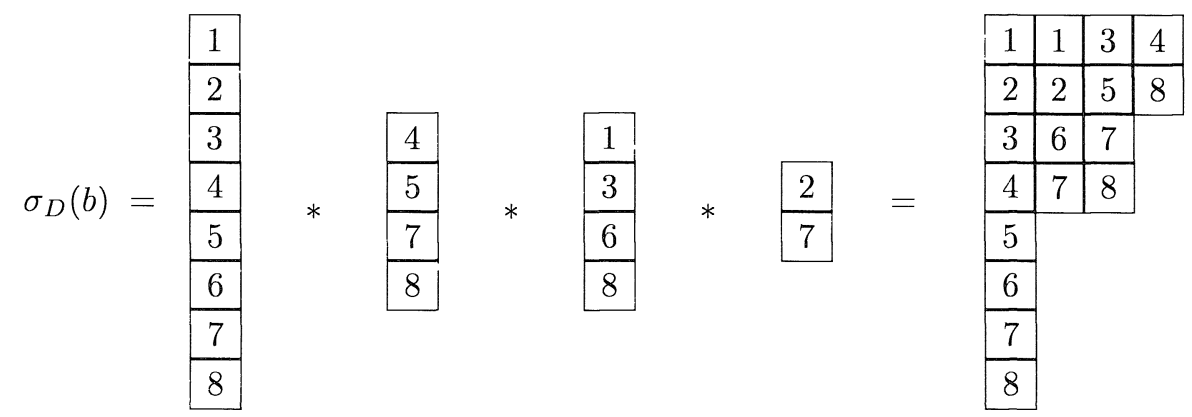


One can now use the automorphism $\sigma_{D}$ to calculate the action of $\tilde{e}_{0}$ and $\tilde{f}_{0}$ on the crystals $B^{n, l}$ and $B^{n-1, l}$, by means of $\tilde{e}_{0}=\sigma_{D}^{-1} \tilde{e}_{n} \sigma_{D}$, and similarly for $\tilde{f}_{0}$.

\section{§2.3. $\mathbb{D}_{n+1}^{(2)}$}

In this subsection, we consider the case of crystals $B^{n, l}$ of type $D_{n+1}^{(2)}$, which are isomorphic to the $B_{n}$ crystals $B\left(l \Lambda_{n}\right)$ when considered as a classical crystal. It turns out that in this case, we can use either of the approaches of the previous 2 sections: use the embedding $B_{n} \hookrightarrow A_{2 n-1}$ and compute the Dynkin automorphism via the promotion operator; use the embedding $B\left(l \Lambda_{n}\right) \hookrightarrow B\left(\Lambda_{n}\right)^{\otimes l}$ of $U_{q}\left(B_{n}\right)$ crystals and compute the automorphism directly. Comparison of these 2 methods leads to an interesting combinatorial identity.

Let us begin with the embedding approach. As for in Section 2.1, consider $A_{2 n-1}$ tableaux on the alphabet $(2.1)$ and let $B_{A}\left(\lambda_{A}\right)$ be the $U_{q}\left(A_{2 n-1}\right)$ crystal with highest weight

$$
\lambda_{A}=\sum_{\imath=1}^{n-1} m_{\imath}\left(\Lambda_{\imath}+\Lambda_{\imath+1}\right)+m_{n} \Lambda_{n}
$$

Then by defining the crystal operators

$$
S\left(\tilde{f}_{\imath}^{(B)}\right)=\left\{\begin{array}{cc}
\tilde{f}_{\imath}^{(A)} \tilde{f}_{\imath+1}^{(A)} & 1 \leq i<n \\
\tilde{f}_{n}^{(A)} & i=n
\end{array}\right.
$$

the crystal obtained by acting with the lowering operators $S\left(\tilde{f}_{\imath}^{(B)}\right)$ on the highest weight element $u_{A}$ of the $U_{q}\left(A_{2 n-1}\right)$ crystal $B_{A}\left(\lambda_{A}\right)$ is isomorphic to the $U_{q}\left(B_{n}\right)$ crystal $B\left(\lambda_{B}\right)$ with highest weight $\lambda_{B}=\sum_{\imath=1}^{n} m_{\imath} \Lambda_{\imath}$ [Kas]. As for the $C_{n}$ case, we shall describe this embedding explicitly.

Let us begin with the case of the crystals $B\left(\omega_{k}\right)$ of the fundamental tensor representations of $U_{q}\left(B_{n}\right)$, where $\omega_{k}=\Lambda_{k}$ for $1 \leq k \leq n-1$ and $\omega_{n}=2 \Lambda_{n}$. The elements of such crystals are described by one-column tableaux of length $k$ on the alphabet

$$
1<2<\cdots<n<0<\bar{n}<\cdots<\overline{2}<\overline{1}
$$

subject to certain conditions which are described in [KN]. Representing such a crystal element by the column tableau $P$, let $P_{+}, \overline{P_{-}}$and $P_{0}$ denote the set of unbarred, barred and zero elements of $P$ respectively. Define the sets

$$
\begin{aligned}
K & :=\left(P_{+} \cap P_{-}\right) \cup P_{0}, \\
J & :=\max \left\{A \subseteq\left(P_{+} \cup P_{-}\right)^{c}|| A|=| K \mid \text { and } A<K\right\}
\end{aligned}
$$


Here, the partial order $<$ is considered with respect to sets of the alphabet $\{1, \ldots, n, 0\}$ with the order $1<\cdots<n<0$. From these sets, define $Q_{ \pm}:=$ $\left(P_{ \pm} / K\right) \cup J$. Again, we can visualize the construction of $Q_{ \pm}$in terms of particles and holes on a pair of vertical ladders. The position 0 is located above position $n$, and we begin with $\left|P_{0}\right|$ particles at position 0 on both the left and right lines of the ladder. One then proceeds as before, starting with the particles at position 0 , moving them to holes located below, and continuing until all the pairs of particles in the initial configuration have been moved in this way. The final configuration represents the set $Q_{ \pm}$. Note that the set $Q:=Q_{+} \cup \bar{Q}_{-}$ contains no zeroes, and $|Q|=|P|+\left|P_{0}\right|$.

The crystal $B_{B}\left(\Lambda_{n}\right)$ of the spinor representation of $U_{q}\left(B_{n}\right)$ is generated as a set by elements $b_{s p}$ which can represented by one-column tableaux on the alphabet $\{1, \ldots, n, \bar{n}, \ldots, \overline{1}\}$, subject to the condition that the entries are strictly increasing vertically, and the entries $i$ and $\bar{i}$ never appear simultaneously. The action of $\tilde{e}_{l}, \tilde{f}_{\imath}$ is given as in $[\mathrm{KN}]$.

We have the following result, proved in much the same way as Prop. 2.2

Proposition 2.6. Define a map $\psi_{t}: B_{B}\left(\omega_{k}\right) \hookrightarrow B_{A}\left(\Lambda_{k}+\Lambda_{2 n-k}\right)$ by

$$
\psi_{t}(P):=\begin{array}{l|l}
P_{-}^{c} \\
\left(\bar{Q}_{+}\right)^{c}
\end{array} \mid \begin{aligned}
& P_{+} \\
& \bar{Q}_{-}
\end{aligned}
$$

and let $\psi_{s p}: B_{B}\left(\Lambda_{n}\right) \rightarrow B_{A}\left(\Lambda_{n}\right)$ be given by the identity map. Then $\psi_{t}$, and $\psi_{\text {sp }}$ are crystal embeddings under the map (2.12).

Example. For $n=8$, let

$$
P=\begin{array}{|l|}
\hline 2 \\
\hline 4 \\
\hline 7 \\
\hline 0 \\
\hline 0 \\
\hline \overline{7} \\
\hline \overline{4} \\
\hline
\end{array} \quad Q=\begin{array}{|l|l|l|}
\hline 2 \\
\hline 3 \\
\hline 5 \\
\hline 6 \\
\hline 8 \\
\hline \overline{8} \\
\hline \overline{6} \\
\hline \overline{5} \\
\hline \overline{3} \\
\hline
\end{array} \quad \Rightarrow \psi_{t}(P)=\begin{array}{|c|c|}
\hline 1 & 2 \\
\hline 2 & 4 \\
\hline 3 & 7 \\
\hline 5 & \overline{8} \\
\hline 6 & \overline{6} \\
\hline 8 & \overline{5} \\
\hline \overline{7} & \overline{3} \\
\hline \overline{4} & \\
\hline \overline{1} \\
\hline
\end{array}
$$

For the general case, we distinguish between tableaux which form the crystals of tensor representations of $U_{q}\left(B_{n}\right)$ with highest weight

$$
\lambda_{t}=\omega_{m_{1}}+\cdots+\omega_{m_{n}}, \quad m_{1} \leq m_{2} \leq \cdots \leq m_{p}
$$




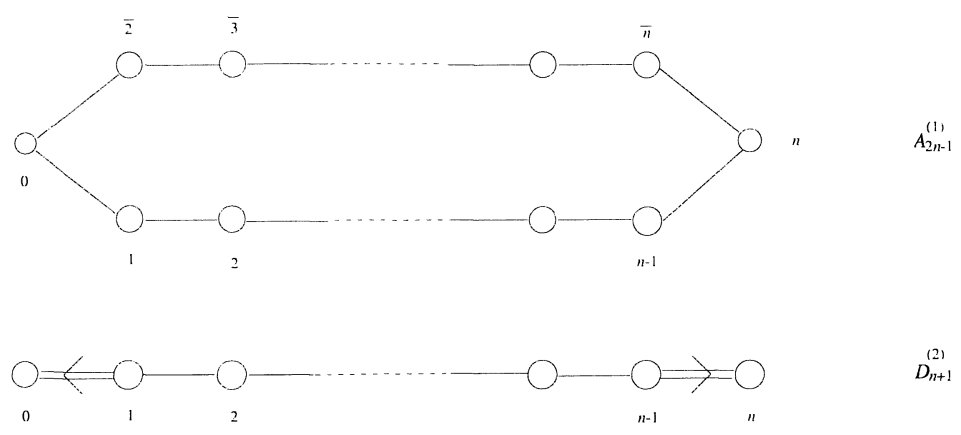

Figure 2.3. Dynkin diagrams for $A_{2 n-1}^{(1)}$ and $D_{n+1}^{(2)}$.

and those of the spinor representations with highest weight

$$
\lambda_{s p}=\omega_{m_{1}}+\cdots+\omega_{m_{p}}+\Lambda_{n}, \quad m_{1} \leq m_{2} \leq \cdots \leq m_{p}
$$

For $b \in B_{B}\left(\lambda_{t}\right)$, (resp. $\left.B_{B}\left(\lambda_{s p}\right)\right)$, write $b=b_{1} \otimes \cdots \otimes b_{p}\left(\otimes b_{s p}\right)$, where $b_{i} \in$ $B_{B}\left(\omega_{m_{i}}\right), b_{s p} \in B_{B}\left(\Lambda_{n}\right)$. Then we have

Proposition 2.7. For $b \in B_{B}\left(\lambda_{t}\right)\left(\right.$ resp. $\left.B_{B}\left(\lambda_{s p}\right)\right)$, define a map $E$ by

$$
E(b):=\psi_{t}\left(b_{1}\right) * \psi_{t}\left(b_{2}\right) * \cdots * \psi_{t}\left(b_{p}\right)\left(* \psi_{s p}\left(b_{s p}\right)\right)
$$

where $*$ is defined in (2.6). Then we have

(i) $E: B_{B}\left(\lambda_{t}\right) \hookrightarrow B_{A}\left(\lambda^{\prime}\right)$ where $\lambda^{\prime}=\sum_{i=1}^{p} \Lambda_{m_{i}}+\Lambda_{2 n-m_{i}}$ (resp. $E: B_{B}\left(\lambda_{s p}\right) \hookrightarrow$ $B_{A}\left(\lambda^{\prime \prime}\right)$ where $\left.\lambda^{\prime \prime}=\sum_{i=1}^{p} \Lambda_{m_{i}}+\Lambda_{2 n-m_{i}}+\Lambda_{n}\right)$ is a crystal embedding.

(ii)

$$
\phi_{i}^{(B)}(b)=\left\{\begin{array}{cc}
\phi_{i}^{(A)}(E(b))=\phi_{i+1}^{(A)}(E(b)) & 1 \leq i<n \\
\phi_{n}^{(A)}(E(b)) & i=n
\end{array}\right.
$$

and similarly for $\varepsilon_{i}^{(B)}(b)$.

Let us now turn to the problem of computing the action of $\tilde{e}_{0}, \tilde{f}_{0}$ for the perfect crystals $B^{n, l}$, which is isomorphic to $B_{B}\left(l \Lambda_{n}\right)$ as a crystal for $B_{n}$. Examination of the Dynkin diagrams of $D_{n+1}^{(2)}$ and $A_{2 n-1}^{(1)}$ reveals that the Dynkin automorphism $\sigma_{B}$ defined on the diagram of $D_{n+1}^{(2)}$ by $\sigma_{B}\left(\alpha_{i}\right)=\alpha_{n-i}$ is related to the $A_{2 n-1}^{(1)}$ automorphism (given by the promotion operator $p r$ ) by $\sigma_{B}=E^{-1}(p r)^{n} E$ and hence the action of $\tilde{f}_{0}$ on this crystal is

$$
S\left(\tilde{f}_{0}\right)=\sigma_{B}^{-1} S\left(\tilde{f}_{n}\right) \sigma_{B}=E^{-1}(p r)^{-1} \tilde{f}_{1}^{(A)} \operatorname{pr} E
$$


and similarly for $S\left(\tilde{e}_{0}\right)$.

For the $B_{n}$ crystal $B_{B}\left(l \Lambda_{n}\right)$, we can consider a different version of the above embedding in the following way. Consider an element $b=b_{1} \otimes \cdots \otimes b_{l} \in$ $B_{B}\left(l \Lambda_{n}\right) \hookrightarrow B_{B}\left(\Lambda_{n}\right)^{\otimes l}$. In other words, $b_{\imath} \in B_{B}\left(\Lambda_{n}\right)$, for each $1 \leq i \leq l$. By writing such an element $b$ as a tableau whose $i$ 'th column (reading from right to left) is given by $b_{\imath}$, then one can show, as in Prop. 2.4 , that the crystal $B_{B}\left(l \Lambda_{n}\right)$ is isomorphic to the set of such tableaux of shape $\left(l^{n}\right)$, whose subtableau of unbarred entries is a valid tableau on the alphabet $\{1, \ldots, n\}$, and where the action of the crystal operators is given by (2.12). One can then compute the action of $\sigma_{B}$ using the promotion operator as before. Alternatively, we have the analog of Prop. 2.5

Proposition 2.8. If $b_{\jmath} \in B_{B}\left(\Lambda_{n}\right)$, let $I=\left(i_{1}, \ldots, i_{p}\right)$ be its column reading of unbarred entries, and let $I^{c}=\left(i_{1}^{\prime}, \ldots, i_{q}^{\prime}\right)$ be the set of barred entries of $b_{\imath}$, without the bars. Defining

$$
\begin{aligned}
\omega\left(b_{\imath}\right) & =\left(n+1-i_{q}^{\prime}, \ldots, n+1-i_{1}^{\prime}\right) \\
\Omega(b) & =\omega\left(b_{1}\right) * \cdots * \omega\left(b_{l}\right)
\end{aligned}
$$

Then $\sigma_{B}(b)$ is the unique tableau whose unbarred subtableau is given by $\Omega(b)$.

Example. Let

$$
b=\begin{array}{|l|l|l|l|}
\hline 1 & 2 & 2 & 3 \\
\hline 2 & 3 & 4 & 5 \\
\hline 4 & 4 & 5 & \overline{6} \\
\hline 5 & 6 & 6 & \overline{4} \\
\hline 6 & \overline{5} & \overline{3} & \overline{2} \\
\hline \overline{3} & \overline{1} & \overline{1} & \overline{1} \\
\hline
\end{array}
$$$$
\sigma_{B}(b)=p r^{6}(b)=\begin{array}{|c|c|c|c|}
\hline 1 & 3 & 5 & 6 \\
\hline 2 & 4 & 6 & \overline{5} \\
\hline 4 & \overline{6} & \overline{4} & \overline{4} \\
\hline 6 & \overline{5} & \overline{3} & \overline{3} \\
\hline \overline{5} & \overline{2} & \overline{2} & \overline{2} \\
\hline \overline{3} & \overline{1} & \overline{1} & \overline{1} \\
\hline
\end{array}
$$

Alternatively,

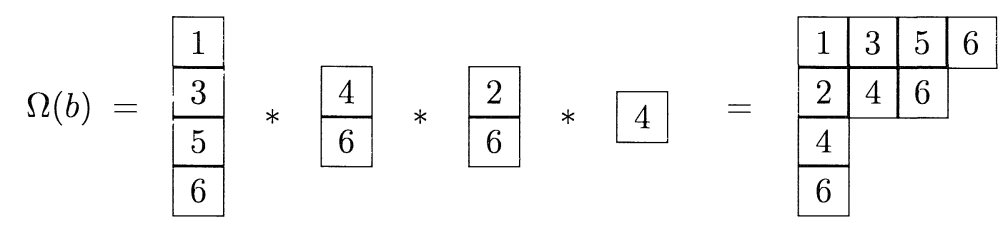


Remark. The fact that $p r^{n}$ and Schensted column insertion are related for this special type of rectangular tableau (where within each column, the barred and unbarred entries are complements of $\{1, \ldots, n\})$ is shown here using crystal base theory. It would be interesting to have a direct combinatorial proof of such a relationship.

\section{§3. Energy Functions}

We now turn to the problem of computing the energy function of a finite, homogeneous path of elements in the crystals mentioned in the introduction. Let us recall the fundamental facts about such energy functions. Suppose $B_{1}$, $B_{2}$ are finite $U_{q}^{\prime}(\mathfrak{g})$ crystals of level $l$ such that $B_{1} \otimes B_{2}$ is connected and $B_{1} \otimes B_{2} \simeq B_{2} \otimes B_{1}$. Suppose that under this isomorphism $b_{1} \otimes b_{2} \mapsto b_{2}^{\prime} \otimes b_{1}^{\prime}$.

It is known [KMN1, HKKOT] that there exists a function (local energy function) $H: B_{1} \times B_{2} \rightarrow \mathbb{Z}$ which is determined (up to an overall additive constant) by the rule

$H\left(\tilde{e}_{2}\left(b_{1} \otimes b_{2}\right)\right)=\left\{\begin{array}{cc}H\left(b_{1} \otimes b_{2}\right)+1 \text { if } i=0, \phi_{0}\left(b_{1}\right) \geq \varepsilon_{0}\left(b_{2}\right), & \phi_{0}\left(b_{2}^{\prime}\right) \geq \varepsilon_{0}\left(b_{1}^{\prime}\right) \\ H\left(b_{1} \otimes b_{2}\right)-1 \text { if } i=0, \phi_{0}\left(b_{1}\right)<\varepsilon_{0}\left(b_{2}\right), & \phi_{0}\left(b_{2}^{\prime}\right)<\varepsilon_{0}\left(b_{1}^{\prime}\right) \\ H\left(b_{1} \otimes b_{2}\right) & \text { otherwise }\end{array}\right.$

Consider the set $\mathcal{P}_{L}$ of finite inhomogeneous paths of length $L$ in the crystal $B_{1} \otimes \cdots \otimes B_{L}$. i.e. $\mathcal{P}_{L}=\left\{p=b_{1} \otimes b_{2} \otimes \cdots \otimes b_{L} \mid b_{\jmath} \in B_{\jmath} 1 \leq j \leq L\right\}$. The crystals $B_{\jmath}$ are such that a local energy function exists on $B_{\jmath} \otimes B_{\jmath+1}$ for each $1 \leq j \leq L-1$. For each $i, 2 \leq i \leq L$, define crystal elements $b_{\imath}^{(2)}, b_{\imath}^{(3)}, \ldots, b_{\imath}^{(\imath)}$ through the successive isomorphisms

$$
b_{1} \otimes \cdots \otimes b_{\imath} \mapsto b_{1} \otimes \cdots \otimes b_{\imath}^{(\imath-1)} \otimes b_{\imath-1}^{\prime} \mapsto \cdots \mapsto b_{1} \otimes b_{\imath}^{(2)} \otimes b_{2}^{\prime} \otimes \cdots \otimes b_{\imath-1}^{\prime}
$$

Using these elements, define

$$
\operatorname{ind}_{p}(i)=\sum_{\jmath=1}^{\iota-1} H\left(b_{\jmath} \otimes b_{\imath}^{(\jmath+1)}\right), \quad E(p)=\sum_{i=2}^{L} \operatorname{ind}_{p}(i)
$$

In the homogeneous case when all $B_{\jmath}$ are equal, $b_{\imath}^{(\jmath+1)}=b_{\jmath+1}$ and the energy becomes 


$$
E(p)=\sum_{j=1}^{L-1}(L-j) H\left(b_{\jmath} \otimes b_{\jmath}+1\right)
$$

Since the local energy function $H$ is constant on connected components of $\mathcal{P}_{L}$, when decomposed as a $U_{q}\left(\mathfrak{g}_{0}\right)$ crystal, it is sufficient to be able to compute $E$ on the $p \in \mathcal{P}$ which are highest weight elements when $\mathcal{P}_{L}$ is considered as a $U_{q}\left(\mathfrak{g}_{0}\right)$ crystal i.e. $p$ is classically admissible.

In the following subsections, we shall give a procedure for associating to each classically admissible path $p$ a semi-standard tableau $T(p)$ for certain level one perfect crystals of $D_{n}^{(1)}, D_{n+1}^{(2)}$ and $C_{n}^{(1)}$. For such combinatorial objects, we can associate a charge such that charge $(T(p))$ is related to $E(p)$ defined in (3.2).

\section{$\S 3.1 . D_{n}^{(1)}$}

In this subsection, we shall compute the local energy function for the $U_{q}^{\prime}\left(D_{n}^{(1)}\right)$ crystals $\left(B^{n, 1}\right)^{\otimes 2}$ and $\left(B^{n-1,1}\right)^{\otimes 2}$, and explicitly describe the decomposition of such crystals regarded as $U_{q}\left(D_{n}\right)$ crystals. Let us recall some notation: let $\omega_{k}=\epsilon_{1}+\cdots+\epsilon_{k}$ for $1 \leq k<n$ and $\omega_{n}^{ \pm}=\epsilon_{1}+\cdots+\epsilon_{n-1} \pm \epsilon_{n}$ be the usual weights for the fundamental tensor representations of $D_{n}$. These are related to the fundamental dominant weights by $\omega_{\imath}=\Lambda_{\imath}, 1 \leq i \leq n-2$, $\omega_{n-1}=\Lambda_{n-1}+\Lambda_{n}, \omega_{n}^{-}=2 \Lambda_{n-1}$ and $\omega_{n}^{+}=2 \Lambda_{n}$. For simplicity we write $B_{+}$ (resp. $B_{-}$) for the $U_{q}\left(D_{n}\right)$ crystal $B\left(\Lambda_{n}\right)$ (resp. $B\left(\Lambda_{n-1}\right)$ ). For an element $b \in B_{ \pm}$, we often write $b=\left[i_{1}, \ldots, i_{p}, \overline{j_{q}}, \ldots, \overline{j_{1}}\right]$ if the "spinor" tableau corresponding to $b$ has column reading $i_{1} \cdots i_{p} \overline{j_{q}} \cdots \overline{j_{1}}$. Moreover, since such an element is uniquely specified by its barred or unbarred entries, we also write $b=\left[i_{1}, \ldots, i_{p}\right]_{+}=\left[\overline{j_{q}}, \ldots, \overline{j_{1}}\right]_{-}$.

Firstly, recall that there is a decomposition of $B_{ \pm} \otimes B_{ \pm}$of the form

$$
B_{ \pm} \otimes B_{ \pm} \simeq B\left(\omega_{n}^{ \pm}\right) \oplus \bigoplus_{k=1}^{[n / 2]} B\left(\omega_{n-2 k}\right)
$$

where $[x]$ denotes the largest integer less than or equal to $x$. In this decomposition, the highest weight element of $B\left(\omega_{k}\right)$ is given for $k \leq n-2$ by

$$
v_{k}^{ \pm}:=\left\{\begin{array}{cl}
{[1,2, \ldots, n] \otimes[1, \ldots, k, \bar{n}, \ldots, \overline{k+1}]} & \text { for }+ \\
{[1,2, \ldots, n-1, \bar{n}] \otimes[1, \ldots, k, n, \overline{n-1}, \ldots, \overline{k+1}]} & \text { for }-
\end{array}\right.
$$


and

$$
v_{n}^{ \pm}:=\left\{\begin{array}{cc}
{[1,2, \ldots, n] \otimes[1, \ldots, n]} & \text { for }+ \\
{[1,2, \ldots, n-1, \bar{n}] \otimes[1, \ldots, n-1, \bar{n}]} & \text { for }-
\end{array}\right.
$$

Using the tensor product rule (1.1) we have for $k \leq n-2$ for + (resp. $k \leq n-4$ for - )

$$
\begin{aligned}
& \left(\tilde{f}_{2} \cdots \tilde{f}_{k+1}\right)\left(\tilde{f}_{1} \cdots \tilde{f}_{k}\right) v_{k}^{ \pm} \\
& =\left\{\begin{array}{cc}
{[1, \ldots, n] \otimes[3, \ldots, k+2, \bar{n}, \ldots, \overline{k+3}, \overline{2}, \overline{1}]=: u_{k}^{+}} & \text {for }+ \\
{[1,2, \ldots, n-1, \bar{n}] \otimes[3, \ldots, k+2, n, \overline{n-1}, \ldots, \overline{k+3}, \overline{2}, \overline{1}]=: u_{k}^{-}} & \text {for }-
\end{array}\right.
\end{aligned}
$$

For the special case of $v_{n-2}^{-}$, we have

$$
\left(\tilde{f}_{2} \cdots \tilde{f}_{n-2}\right) \tilde{f}_{n}\left(\tilde{f}_{1} \cdots \tilde{f}_{n-2}\right) v_{n-2}^{-}=[1, \ldots, n-1, \bar{n}] \otimes[3, \ldots, n-1, \bar{n}, \overline{2} \cdot \overline{1}]=: u_{n-2}^{-}
$$

By the action of $\tilde{f}_{0}$ described in [KMN2], we have $\tilde{f}_{0} u_{k}^{ \pm}=v_{k+2}^{ \pm}$. Thus, if we fix $H$ by setting $H\left(b_{1} \otimes b_{2}\right)=0$ for $b_{1} \otimes b_{2} \in B\left(\omega_{n}^{ \pm}\right)$, we have

$$
H\left(b_{1} \otimes b_{2}\right)=-k \quad \text { if } b_{1} \otimes b_{2} \in B\left(\omega_{n-2 k}\right)
$$

So, given $b_{1} \otimes b_{2} \in B_{ \pm}^{\otimes 2}$, to compute the value of $H$, it is sufficient to determine in which component $b_{1} \otimes b_{2}$ lies in the decomposition (3.3). This can be achieved through the explicit description of (3.3), which we now give. From [KN], the one-column $D_{n}$ tableaux appearing in (3.3) have column reading $\left(u_{1}, \ldots, u_{r}, \alpha_{1}, \ldots, \alpha_{z}, \overline{v_{s}}, \ldots, \overline{v_{1}}\right)$, where $\left(\alpha_{1}, \ldots, \alpha_{z}\right)$ is a sequence of consecutive $n$ 's and $\bar{n}$ 's.

Proposition 3.1. Suppose $b_{1}=\left[\overline{j_{q}}, \ldots, \overline{j_{1}}\right]_{-}$and $b_{2}=\left[i_{1}, \ldots, i_{p}\right]_{+} . B y$ letting $w_{0}:=\left(\overline{j_{q}}, \ldots, \overline{j_{1}}\right)$, define $w_{k}$ by successively inserting $i_{k}$ into $w_{k-1}=$ $\left(u_{1}, \ldots, u_{r}, \alpha_{1}, \ldots, \alpha_{z}, \overline{v_{s}}, \ldots, \overline{v_{1}}\right)$, using the rule 


$$
\begin{aligned}
& \left\{\begin{array}{cr}
\left(u_{1}, \ldots, u_{r}, i_{k}, \alpha_{1}, \ldots, \alpha_{z}, \overline{v_{s}}, \ldots, \overline{v_{1}}\right) & i_{k} \notin\left\{v_{1}, \ldots, v_{s}\right\} \quad\left(R_{1}\right) \\
\left(u_{1}, \ldots, u_{r}, c, \alpha_{1}, \ldots, \alpha_{z}, \overline{v_{s}}, \ldots, \overline{v_{t}}, \bar{c}, \overline{c-1}, \ldots, \overline{i_{k}+1}, \overline{v_{l}}, \ldots, \overline{v_{1}}\right) & \\
\text { if } w_{k-1}=\left(u_{1}, \ldots, u_{r}, \alpha_{1}, \ldots, \alpha_{z}, \overline{v_{s}}, \ldots, \overline{v_{t}}, \overline{c-1},\right. \\
\left.\ldots, \overline{i_{k}+1}, \overline{i_{k}}, \overline{v_{l}}, \ldots, \overline{v_{1}}\right) \quad\left(R_{2}\right)
\end{array}\right. \\
& \left(u_{1}, \ldots, u_{r}, n, \alpha_{1}, \ldots, \alpha_{z}, \bar{n}, \ldots, \overline{i_{k}+1}, \overline{v_{l}}, \ldots, \overline{v_{1}}\right) \\
& \text { if } \quad w_{k-1}=\left(u_{1}, \ldots, u_{r}, \alpha_{1}, \ldots, \alpha_{z-1}, \bar{n}, \ldots, \overline{i_{k}+1}, \overline{i_{k}}, \overline{v_{l}}, \ldots, \overline{v_{1}}\right) \text {, } \\
& w_{k}=\left\{\begin{array}{cc}
\alpha_{1}=\bar{n} \quad\left(R_{3}\right) \\
\left(u_{1}, \ldots, u_{r}, \bar{n}, \alpha_{1}, \ldots, \alpha_{z-1}, \bar{n}, \ldots, \overline{i_{k}+1}, \overline{v_{l}}, \ldots, \overline{v_{1}}\right) & \\
\text { if } w_{k-1}=\left(u_{1}, \ldots, u_{r}, \alpha_{1}, \ldots, \alpha_{z-1}, \bar{n}, \ldots, \overline{i_{k}+1}, \overline{i_{k}}, \overline{v_{l}}, \ldots, \overline{v_{1}}\right),
\end{array}\right. \\
& \alpha_{1}=n \quad\left(R_{4}\right) \\
& \begin{array}{l}
\left(u_{1}, \ldots, u_{r}, \alpha_{1}, \ldots, \alpha_{z-1}, \bar{n}, \ldots, \overline{i_{k}+1}, \overline{v_{l}}, \ldots, \overline{v_{1}}\right) \\
\quad \text { if } \quad w_{k-1}=\left(u_{1}, \ldots, u_{r}, \alpha_{1}, \ldots, \alpha_{z-1}, \bar{n}, \ldots, \overline{i_{k}+1}\right.
\end{array} \\
& \left.\overline{i_{k}}, \overline{v_{l}}, \ldots, \overline{v_{1}}\right), \quad\left(R_{5}\right) \\
& k=p, \quad n \notin J, z \text { even, } \text { or } n \in J, z \text { odd }
\end{aligned}
$$

where $J=\left\{j_{1}, \ldots, j_{q}\right\}$. Then the isomorphism (3.3) is given by $b_{1} \otimes b_{2} \mapsto w_{p}$.

Proof. First note that $w t\left(b_{1} \otimes b_{2}\right)=w t\left(w_{p}\right)$ (the last case in the definition of $w_{p}$ ensures that $\left\langle w t\left(b_{1} \otimes b_{2}\right), \epsilon_{n}\right\rangle=\left\langle w t\left(w_{p}\right), \epsilon_{n}\right\rangle$.) To show that the above map respects the tensor product rule (1.1), one must consider for each $i, 1 \leq i \leq n-1$ the 16 possible cases according to the presence or absence of $i, i+1$ in the sets $I$ and $J$. We omit the details. Finally, to show such a map is a bijection, one must show it's invertible. To this end, consider the set $D_{p}:=\left(u_{1}, \ldots, u_{r}, \alpha_{1}, \ldots, \alpha_{y}\right)$ of all elements strictly less than $\overline{n-1}$ in $w_{p}$. By checking the 4 cases according to whether $n$ or $\bar{n}$ belongs to $b_{1}, b_{2}$, the last insertion (of $i_{p}$ ) was an $\left(R_{5}\right)$ insertion iff " $\left|D_{p}\right|$ is even (resp. odd) and $\alpha_{y}=\bar{n}$ " or " $\left|D_{p}\right|$ is odd (resp. even) and $\alpha_{y}=n$ " in the cases $n$ even, $B_{+}$or $n$ odd, $B_{-}$(resp. $n$ odd, $B_{+}$or $n$ even, $\left.B_{-}\right)$.

Set $I_{p}:=\{\}$. If the last insertion was done by $\left(R_{5}\right)$, then $i_{p}$ must be the largest barred integer $\bar{s}$ say, not appearing in $w_{p}$. Thus, let $I_{p}:=\{s\}$, $w_{p}=w_{p} \cup\{\bar{s}\}$. Hence or otherwise, for each $\alpha_{\imath} \in D_{p}$ for $1 \leq i \leq y$, do

1. $D_{p-\imath}:=D_{p-\imath+1} /\left\{\alpha_{\imath}\right\}$, 
2. $I:=I \cup\{s\}$, where $\bar{s}$ is the largest (as unbarred entry) integer not appearing in $w_{p-\imath+1}$

3. $w_{p-\imath}:=w_{p-\imath+1} /\left\{\alpha_{\imath}\right\} \cup\{\bar{s}\}$

This reverses the insertions by $\left(R_{3}\right)$ and $\left(R_{4}\right)$. To reverse the remaining insertions by $\left(R_{1}\right)$ and $\left(R_{2}\right)$, then for each $u_{r+1-\imath} \in D_{p-y}, 1 \leq i \leq r$ do

1. if $\overline{u_{r+1-\imath}} \notin w_{p-y-\imath+1}$, then $D_{p-y-\imath}:=D_{p-y-\imath+1} /\left\{u_{r+1-\imath}\right\}, w_{p-y-\imath}:=$ $w_{p-y-\imath+1} /\left\{u_{r+1-\imath}\right\}, I:=I \cup\left\{u_{r+1-\imath}\right\}$

2. if $\overline{u_{r+1-\imath}} \in w_{p-y-\imath+1}$, then $D_{p-y-\imath}:=D_{p-y-\imath+1} /\left\{u_{r+1-\imath}\right\}, w_{p-y-\imath}:=$ $w_{p-y-\imath+1} /\left\{u_{r+1-\imath}\right\}, I:=I /\left\{\overline{u_{r+1-\imath}}\right\} \cup\{\bar{s}\}$, where $\bar{s}$ is the largest (as unbarred entry) integer $<u_{r+1-\imath}$ not appearing in $w_{p-\imath+1}$

Example. For $n=10$, let

$$
b_{1}=[1,3,4,8, \overline{10}, \overline{9}, \overline{7}, \overline{6} . \overline{5}, \overline{2}], \quad b_{2}=[3,6,7,10, \overline{9}, \overline{8}, \overline{5}, \overline{4}, \overline{2}, \overline{1}]
$$

Then

$$
\begin{array}{r}
(\overline{10}, \overline{9}, \overline{7}, \overline{6}, \overline{5}, \overline{2}) \stackrel{3}{\rightarrow}(3, \overline{10}, \overline{9}, \overline{7}, \overline{6}, \overline{5}, \overline{2}) \stackrel{6}{\rightarrow}(3,8, \overline{10}, \overline{9}, \overline{8}, \overline{7}, \overline{5}, \overline{2}) \\
\stackrel{7}{\rightarrow}(3,8,10, \overline{10}, \overline{9}, \overline{8}, \overline{5}, \overline{2}) \stackrel{10}{\rightarrow}(3,8, \overline{10}, 10, \overline{9}, \overline{8}, \overline{5}, \overline{2})
\end{array}
$$

Thus, given $b_{1} \otimes b_{2} \in B_{ \pm}^{\otimes 2}$, the quantity $H\left(b_{1} \otimes b_{2}\right)$ is simply $\left(\left|w_{p}\right|-\right.$ $n) / 2$. Let us view this in terms of a "winding diagram" in the spirit of Nakayashiki and Yamada [NY]. Consider two parallel vertical ladders with the positions $1, \ldots, n, \bar{n}, \ldots, \overline{1}$ labeled from top to bottom. Given $b_{1} \otimes b_{2} \in$ $B_{ \pm}^{\otimes 2}$, place particles at positions $i_{1}, \ldots, i_{p}, \overline{j_{q}}, \ldots, \overline{j_{1}}$ on the left ladder, if $b_{1}=\left[i_{1}, \ldots, i_{p}, \overline{j_{q}}, \ldots, \overline{j_{1}}\right]$, and likewise for $b_{2}$. Starting with the topmost particle $s$ of $b_{2}$, locate the first particle in $b_{1}$ whose position is lower than or equal to $s$, which is not connected by a line. If no such particle exists, locate the topmost unconnected particle in $b_{1}$. Connect these two particles by a line. Call such a line a "up" $\mathrm{H}$-line if the particle in $b_{1}$ is lower or equal than the particle in $b_{2}$, a "down" (or winding) H-line if it is higher. Repeat this procedure for the remaining particles in $b_{2}$, in descending order. Call such a diagram the $H$-diagram of $b_{1} \otimes b_{2}$. It was shown in [NY] (in a much more general situation) that the number of down $H$-lines is independent of the order you connect the particles. 
Proposition 3.2. Let $u$ be the number of winding H-lines and let $c$ denote the number of particles $x \in b_{2}$ which are connected to a particle $\bar{y} \in b_{1}$. Then

$$
-2 H\left(b_{1} \otimes b_{2}\right)=u+\delta(c \in 2 \mathbb{Z}+1)
$$

where $\dot{\delta}(\backslash)=1$ if $\chi$ is true, and 0 is $\backslash$ is false.

Proof. Suppose $b_{1}=\left[i_{1}, \ldots, i_{p}, \overline{j_{q}}, \ldots, \overline{j_{1}}\right]$ and $b_{2}=\left[l_{1}, \ldots, l_{p^{\prime}}, \overline{m_{q^{\prime}}}, \ldots, \overline{m_{1}}\right]$. Then if we compute the image of $b_{1} \otimes b_{2}$ using the rules $\left(R_{1}\right)-\left(R_{5}\right)$, we have

$$
\left|w_{p^{\prime}}\right|=q+\mid\left\{k \mid l_{k} \text { inserted using } R_{1} \text { or } R_{2}\right\} \mid-\delta\left(l_{p^{\prime}} \text { inserted using } R_{5}\right) .
$$

Examining the rules $\left(R_{1}\right)$ and $\left(R_{2}\right)$ carefully, we see that $l_{k}$ is inserted using $\left(R_{1}\right)$ or $\left(R_{2}\right)$ iff there is an up line connecting $l_{k} \in b_{2}$ with some $i_{s} \in b_{1}$. It follows from Lemma A.1 (but applied to H-diagrams instead of LS-diagrams) that the only winding lines in the diagram of $b_{1} \otimes b_{2}$ which may occur emanate from particles in $\left\{\overline{m_{q^{\prime}}}, \ldots, \overline{m_{1}}\right\}$ and connect to particles in $\left\{i_{1}, \ldots, i_{p}\right\}$. Let $f$ be the number of such winding lines, and let $d$ be the number of up lines connecting particles in $\left\{l_{1}, \ldots, l_{p^{\prime}}\right\}$ to $\left\{i_{1}, \ldots, i_{p}\right\}$. Then certainly $p=d+f$. Since $p+q=n$, we have

$$
\left|w_{p^{\prime}}\right|=q+d-\delta\left(l_{p^{\prime}} \text { inserted using } R_{5}\right)=n-f-\delta\left(l_{p^{\prime}} \text { inserted using } R_{5}\right)
$$

Thus the stated formula (3.5) will follow if we can show the last term in the above equation is equal to $\delta(c \in 2 \mathbb{Z}+1)$. This follows from Prop. 3.1 by checking the 4 cases according to whether $n$ or $\bar{n}$ belong to $b_{1}$ or $b_{2}$.

We remark here that Okado [Oka] has previously given a formula for the value of $H$ on the crystals of the spinor representations of $U_{q}\left(B_{n}\right)$ and $U_{q}\left(D_{n}\right)$, derived by taking the $q \rightarrow 0$ limit of the corresponding $R$-matrices.

Since the value of the local energy function $H\left(b_{1} \circlearrowleft b_{2}\right)$ is related (up to a factor of -2 and possible addition of 1 ) to the number of winding lines in the $\mathrm{H}$-diagram of $b_{1} \otimes b_{2}$, it suggests we can modify the definition of the charge of Lascoux and Schützenberger to give a direct relationship between charge and energy for these level $1 D_{n}^{(1)}$ crystals.

To this end, we first set up a bijection between $D_{n}$ highest weight vectors in $\mathcal{P}_{L}$ and certain semi-standard tableaux of content $\left(n^{L}\right)$. Recall [Nak] the criterion for a vector $b \otimes b_{1} \otimes \cdots \otimes b_{L}$ to be a $D_{n}$ highest weight vector, where each $b_{1} \in B_{ \pm}$. If $b$ is a highest weight vector, one can associate a generalized partition $\lambda^{s}=\left(\lambda_{1}^{s}, \ldots, \lambda_{n}^{s}\right)$ where each $\lambda, \in \frac{1}{2} \mathbb{Z}_{+}$for $1 \leq i<n, \lambda_{n} \in \frac{1}{2} \mathbb{Z}_{\text {and }} \lambda_{1}^{s} \geq \cdots \geq$ 
$\lambda_{n-1}^{s} \geq\left|\lambda_{n}^{s}\right|$. If $b_{1} \in B_{ \pm}$then $b \otimes b_{1}$ is a $D_{n}$ highest weight element iff $\lambda^{s}-\varepsilon\left(b_{1}\right)$ is a dominant weight. Here $\varepsilon(b)=\sum_{\imath=1}^{n} \varepsilon_{\imath}(b) \Lambda_{\imath}$. Similarly $b \otimes b_{1} \otimes \cdots \otimes b_{L}$ is a highest weight element iff $\lambda^{s}+w t\left(b_{1}\right)+\cdots+w t\left(b_{\imath-1}\right)-\varepsilon\left(b_{\imath}\right)$ is a dominant weight for each $1 \leq i \leq L$. This then implies that $\lambda^{s}+w t\left(b_{1}\right)+\cdots+w t\left(b_{\imath}\right)$ is a generalized partition for each $1 \leq i \leq L$.

Example. Consider the highest weight element

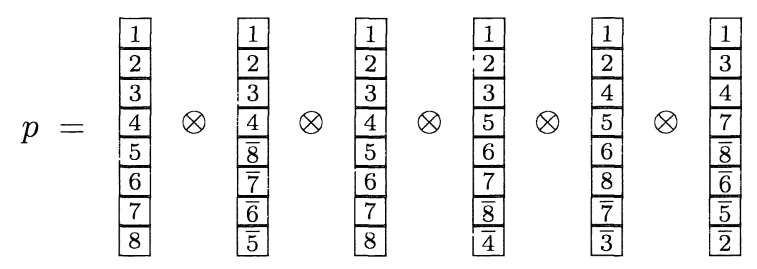

This corresponds to the sequence of shape changes
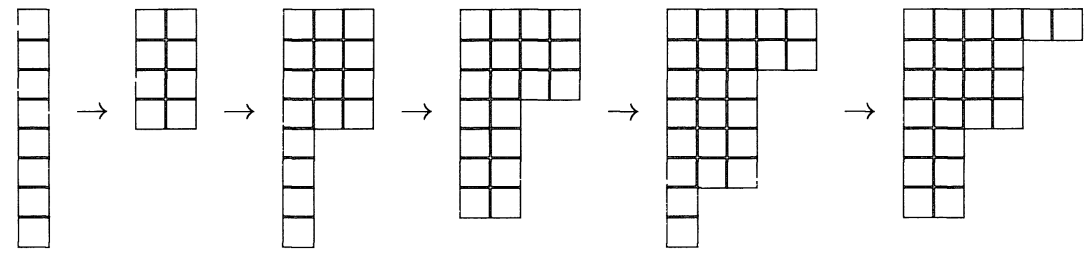

Here, each square represents a "half-box", so that the final shape represents the partition (3222111).

Alternatively, given a highest weight element $p \in \mathcal{P}_{L}$ we can associate a semi-standard tableau $T(p)$ of shape $\nu=\lambda+\mu$ recursively as follows. If $p^{\prime} \in \mathcal{P}_{L-1}$ is given, and $p=p^{\prime} \otimes b_{L}$, then for each $i_{k} \in b_{L}$, place the entry $L$ in the $i_{k}$ 'th column of $T\left(p^{\prime}\right)$, and for each $\overline{j_{l}} \in b_{L}$, place the entry $L$ in the $\left(2 n+1-j_{l}\right)^{\prime}$ th column of $p^{\prime}$. It is clear that the shape $\lambda$ of the subtableau in the first $n$ columns, and the shape $\mu$ of the subtableau in the second $n$ columns are complements in the shape $\left(n^{L}\right)$. Indeed, their conjugates are also complementary partitions in $\left(L^{n}\right)$ and thus related by

$$
\mu_{\imath}^{\prime}=L-\lambda_{n+1-\imath}^{\prime}, \quad 1 \leq i \leq n
$$

The tableau $T(p)$ has content $\left(L^{n}\right)$ and is distinguished by the fact that for each $i$ with $1 \leq i \leq L$, the entry $i$ occur either in column $j$ or column $2 n+1-j$ for each $1 \leq j \leq n$. In other words, the $j$ 'th and $(2 n+1-j)^{\prime}$ th columns are complements in $\{1, \ldots, L\}$. Hence the tableau $T(p)$ is uniquely specified by the subtableaux on the shape $\lambda^{\prime}$. Moreover, if $p \in \mathcal{P}_{L}$ is associated with a 
generalized partition $\lambda^{s}$ as discussed above, then it follows by a simple induction on $L$ that

$$
\lambda^{\prime}=\lambda^{s}+L / 2
$$

Example. For the example given above, we have the tableau

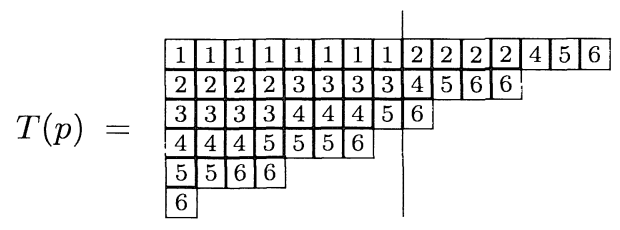

Here, we have $\lambda^{s}=(322111), \lambda^{\prime}=(65554443)$ and $\mu^{\prime}=(3222111)$.

Similar to the notion of H-diagrams, Nakayashiki and Yamada introduced the concept of LS-diagrams, which are related to the computation of the LascouxSchützenberger charge of a semi-standard tableau. Again, one represents the elements of $b_{1}$ and $b_{2}$ as particles on a ladder. Given a particle $x \in b_{1}$ one selects the first unconnected particle $y \in b_{2}$ which is greater than or equal to $x$, is such a particle exists and connects them with an "up" LS-line. If no such particle exists, connect $x$ with the lowest unconnected particle in $b_{2}$, calling such a line, a "down" LS-line. The LS-diagrams of $B_{ \pm}^{\otimes 2}$ have the following properties, proved in Appendix A.

Proposition 3.3. Given an H-diagram (with top-to-bottom order) $H$ of $b_{1} \otimes b_{2} \in B_{ \pm}^{\otimes 2}$ and an LS-diagram (with order $J$ ) L of $b_{1} \otimes b_{2}$ we have

(i) there exists at least 1 order $J$ such that the diagram $L$ coincides with the diagram $H$.

(ii) the number of down LS-lines in $L$ is independent of the order $J$.

(iii) the number of up LS-lines connecting $\bar{x} \in b_{1}$ with $y \in b_{2}$ is independent of the order $J$.

Remark. From the definitions of $\mathrm{H}$ - and LS-diagrams, it is clear that the LS-diagram of $b_{1} \otimes b_{2}$ is identical to the H-diagram of $b_{1}^{\prime} \otimes b_{2}^{\prime}$ obtained by rotating through 180 degrees, where if $b_{1}=\left[i_{1}, \ldots, i_{p}, \overline{j_{q}}, \ldots, \overline{j_{1}}\right], b_{2}=$ $\left[k_{1}, \ldots, k_{r}, \overline{l_{s}}, \ldots, \overline{l_{1}}\right]$, then $b_{1}^{\prime}=\left[l_{1}, \ldots, l_{s}, \overline{k_{r}}, \ldots, \overline{k_{1}}\right], b_{2}^{\prime}=\left[j_{1}, \ldots, j_{q}, \overline{i_{p}}, \ldots, \overline{i_{1}}\right]$. Hence, for any statement about LS-diagrams there is an equivalent statement about H-diagrams. 
Following Nakayashiki and Yamada, we define the local index $\operatorname{ind}_{J}\left(b_{1} \otimes b_{2}\right)$ of the LS-diagram $b_{1} \otimes b_{2}$ with respect to the order $J$ as being the quantity given by the RHS of (3.5). From Prop. 3.3 we have ind ${ }_{J}\left(b_{1} \otimes b_{2}\right)=-2 H\left(b_{1} \otimes b_{2}\right)$. For a path $p=b_{1} \otimes b_{2} \otimes \cdots \otimes b_{L} \in \mathcal{P}_{L}$, drawing the LS lines in the diagram of $b_{1} \otimes b_{2}$ with order $J_{1}$ say, induces an order $J_{2}$ on the LS diagram of $b_{2} \otimes b_{3}$ and so on. For the initial order $J_{1}$, we take the bottom-to-top order (to be consistent with the definition of LS charge). Define

$$
\operatorname{ind}(k)=\operatorname{ind}_{J_{1}}\left(b_{1} \otimes b_{2}\right)+\cdots+\operatorname{ind}_{J_{h-1}}\left(b_{k-1} \otimes b_{k}\right)
$$

Then

$$
-2 E(p)=\sum_{k=1}^{L} \operatorname{ind}(k)
$$

We now define a "local index" and "charge" for the tableau $T(p)$ consistent with the above definitions. Introduce a set of auxiliary counters $v_{\imath}, 1 \leq i \leq$ $L-1$, initialized to 0 . In the usual manner [Mac], attach subscripts to the entries of $T(p)$ recursively as follows: begin with the rightmost entry 1 in $T(p)$ and attach the subscript 0 . Given an entry $i$ with subscript $c$, search the tableau along the rows, using the order right-to-left and top-to-bottom (the Hebrew/Arabic order) until the first entry $i+1$ is encountered. If $i+1$ is encountered before reaching the beginning of the bottom row (i.e. $i+1$ is strictly south and weakly west of $i$ in the tableau $T(p)$ ), then attach $c$ as the subscript of $i+1$. Otherwise, wind around to the end of the first row and continue the search until the first $i+1$ is encountered (such $i+1$ will be located weakly north and strictly east of $i$ ), attaching the subscript $c+1$. If $i$ is located among columns $n+1, \ldots, 2 n$ and $i+1$ is located among columns $1, \ldots, n$, let $v_{\imath} \mapsto v_{\imath}+1$. Continue this process for each $i, 1 \leq i \leq L$. Then repeat the procedure ignoring all entries with subscripts. The local index $\operatorname{ind}^{\prime}(k)$ is just the sum of all the subscripts attached to the entries $k$, and we define

$$
\operatorname{ind}_{T(p)}(k)=\operatorname{ind}^{\prime}(k)+\sum_{\jmath=1}^{k-1} \delta\left(v_{\jmath} \in 2 \mathbb{Z}+1\right)
$$

The quantities $v_{\jmath}$ just count the number of up lines from barred to unbarred elements in the LS diagram of $b_{\jmath} \otimes b_{\jmath+1}$. Finally, define the charge

$$
c(p)=\sum_{k=1}^{L} \operatorname{ind}_{T(p)}(k)
$$

From Prop. 3.3, we have $\operatorname{ind}_{T(p)}(k)=\operatorname{ind}(k)$ and hence $c(p)=-2 E(p)$. 
Example. Continuing with the running example, we have the following values of $-2 H\left(b_{J} \otimes b_{\jmath}+1\right)$ for $1 \leq j \leq 5:(4,0,2,1+1,2)$. Here $1+1$ indicates that $u=1$ and $c \in 2 \mathbb{Z}+1$ in (3.5). Hence

$$
\operatorname{ind}(1)=0 \quad \operatorname{ind}(2)=4 \quad \operatorname{ind}(3)=4 \quad \operatorname{ind}(4)=6 \quad \operatorname{ind}(5)=8 \quad \operatorname{ind}(6)=10
$$

Computing the local index of $T(p)$ we have

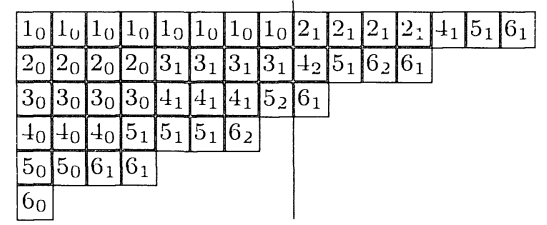

$$
\begin{array}{ccc}
\operatorname{ind}^{\prime}(1)=0 & \operatorname{ind}^{\prime}(2)=4 & \operatorname{ind}^{\prime}(3)=4 \\
\operatorname{ind}^{\prime}(4)=6 & \operatorname{ind}^{\prime}(5)=7 & \operatorname{ind}^{\prime}(6)=9 \\
\left(v_{1}, \ldots,\right. & \left.v_{6}\right)=(0,4,0,1,0,0)
\end{array}
$$

Hence $\operatorname{ind}_{T(p)}(k)=\operatorname{ind}(k)$ and $c(p)=-2 E(p)=32$.

$$
\text { §3.2. } \mathbb{D}_{n+1}^{(2)}
$$

In this subsection, we repeat the analysis of the previous section for the $D_{n+1}^{(2)}$ perfect crystals. Let $\omega_{k}=\epsilon_{1}+\cdots+\epsilon_{k}$ be the weights of the fundamental tensor representations of $B_{n}$, which are related to the fundamental dominant weights by $\omega_{1}=\Lambda_{1}$ for $1 \leq i \leq n-1$ and $\omega_{n}=2 \Lambda_{n}$. We begin with the decomposition of the $B_{n}$ crystal

$$
B\left(\Lambda_{n}\right) \otimes B\left(\Lambda_{n}\right) \simeq \bigoplus_{k=0}^{n} B\left(\omega_{n-k}\right)
$$

The highest weight element of $B\left(\omega_{k}\right)$ is given by $v_{k}:=[1,2, \ldots, n] \otimes[1, \ldots, k, \bar{n}$, $\ldots, \overline{k+1}]$. From the rule (1.1) we have for $k \leq n-1$,

$$
\tilde{f}_{1} \cdots \tilde{f}_{k} v_{k}=[1,2, \ldots, n] \otimes[2, \ldots, k+1, \bar{n}, \ldots, \overline{k+2}, \overline{1}]=: u_{k}
$$

Using the action of the $D_{n+1}^{(2)} \tilde{f}_{0}$ operator [KMN2], we have $\tilde{f}_{0} u_{k}=v_{k+1}$, and hence we have

$$
H\left(b_{1} \otimes b_{2}\right)=-k, \quad \text { if } b_{1} \otimes b_{2} \in B\left(\omega_{n-k}\right)
$$

where we have normalized the energy function such that $H\left(b_{1} \otimes b_{2}\right)=0$ if $b_{1}=b_{2}=[1, \ldots, n]$.

To proceed further, we recall the $B_{n}$ analogue of Prop. 3.1 which was given in $[$ Bak1]. 
Proposition 3.4. Suppose $b_{1}=\left[\overline{j_{q}}, \ldots, \overline{j_{1}}\right]_{-}$and $b_{2}=\left[i_{1}, \ldots, i_{p}\right]_{+}$. Let $u_{0}:=\left(\overline{j_{q}}, \ldots, \overline{j_{1}}\right)$ and given $u_{k-1}=\left(v_{1}, \ldots, v_{l}, \overline{w_{m}}, \ldots, \overline{w_{1}}\right)$, define $u_{k}$, $1 \leq k \leq p$ inductively as

$u_{k}=\left\{\begin{array}{l}\left(v_{1}, \ldots, v_{l}, i_{k}, \overline{w_{m}}, \ldots, \overline{w_{1}}\right) \quad \text { if } i_{k} \notin\left\{w_{1}, \ldots, w_{m}\right\}, \\ \left(v_{1}, \ldots, v_{l}, c, \overline{w_{m}}, \ldots, \overline{w_{s+1}}, \bar{c}, \overline{w_{s-1}}, \ldots, \overline{w_{r+1}}, \overline{w_{r-1}}, \ldots,\right) \\ i_{k}=w_{r}, w_{r+\jmath}=w_{r}+j, 1 \leq j \leq s-r, w_{s+1}>c=w_{s}+1 \\ \left(v_{1}, \ldots, v_{l}, 0, \overline{w_{m}}, \ldots, \overline{w_{r+1}}, \overline{w_{r-1}}, \ldots\right) \\ i_{k}=w_{r}, w_{r+\jmath}=n-m+r+j, 1 \leq j \leq m-r\end{array}\right.$

Then $b_{1} \otimes b_{2} \mapsto u_{p}$ is the crystal isomorphism which realizes (3.7).

It follows that for $b_{1} \otimes b_{2} \in B\left(\Lambda_{n}\right)^{\otimes 2}$, we have $H\left(b_{1} \otimes b_{2}\right)=\left|u_{p}\right|-n$, where $u_{p}$ is given by the algorithm above. Using Prop. 3.4, we have analogously to (3.5), the result

$$
-H\left(b_{1} \otimes b_{2}\right)=u
$$

where $u$ is the number of down lines in the H-diagram of $b_{1} \otimes b_{2}$.

As in the $D_{n}^{(1)}$ case, $p=b \otimes b_{1} \otimes \cdots \otimes b_{L}$ is a $U_{q}\left(B_{n}\right)$ highest weight element iff $\lambda^{s}+w t\left(b_{1}\right)+\cdots+w t\left(b_{\imath-1}\right)-\varepsilon\left(b_{\imath}\right)$ is dominant for each $1 \leq i \leq L$, where $\lambda^{s}=w t(b)$ is the generalized partition associated to the highest weight element $b$.

To such a highest weight element we can associate a semi-standard tableau $\mathrm{T}(\mathrm{p})$ in exactly the same manner as in the $D_{n}^{(1)}$ case. The only difference will be that in the subtableau formed by the first $n$ columns, the number of entries equal to $i$ for any $1 \leq i \leq L$ is not restricted to being even/odd.

Using (3.9) we then have the result that $c(T(p))=-E(p)$ where $c(T(p))=$ $\sum_{k=1}^{L} \operatorname{ind}^{\prime}(k)$, with the local indices $\operatorname{ind}^{\prime}(k)$ being defined as in the $D_{n}^{(1)}$ case.

\section{§3.3. $\mathbb{C}_{n}^{(1)}$}

Finally, we turn to the case of crystals of $U_{q}^{\prime}\left(C_{n}^{(1)}\right)$ studying the energy functions associated to highest weight paths in the space $B^{\otimes L}$, where $B=B^{n, 1}$. This crystal is part of a family of level 1 crystals $B^{r, 1}$ introduced in [HKOTY]. As $U_{q}\left(C_{n}\right)$ crystals, they are isomorphic to the crystal $B\left(\Lambda_{r}\right)$ and hence the elements of such a crystal can be represented by one-column tableaux of length $r$. These crystals are not perfect, unless $r=n$. For $1 \leq i \leq n$, the action of $\tilde{e}_{\imath}$, 
$\tilde{f}_{\imath}$ are given in $[\mathrm{KN}]$, while for $i=0$, we have

$$
\begin{aligned}
& \tilde{f}_{0}\left(i_{1}, \ldots, i_{r}\right)=\left\{\begin{array}{cc}
\left(1, i_{1}, \ldots, i_{r-1}\right) & \text { if } i_{r}=\overline{1} \\
0 & \text { otherwise }
\end{array}\right. \\
& \tilde{e}_{0}\left(i_{1}, \ldots, i_{r}\right)=\left\{\begin{array}{cc}
\left(i_{2}, \ldots, i_{r}, \overline{1}\right) & \text { if } i_{1}=1 \\
0 & \text { otherwise }
\end{array}\right.
\end{aligned}
$$

To compute the value of the local energy function $H$ on $B^{r_{1}, 1} \otimes B^{r_{2}, 1}$, let us first decompose this as a $U_{q}\left(C_{n}\right)$ crystal

$$
B\left(\Lambda_{r_{1}}\right) \otimes B\left(\Lambda_{r_{2}}\right) \simeq \underset{\substack{0 \leq s \leq t \leq n \\ s+t \leq r_{1}+r_{2}, s+t \equiv r_{1}+r_{2}(\bmod 2) \\\left|r_{1}-r_{2}\right| \leq t-s \leq 2 n-r_{1}-r_{2}}}{\bigoplus} B\left(\Lambda_{t}+\Lambda_{s}\right)
$$

The highest weight element of $B\left(\Lambda_{t}+\Lambda_{s}\right)$ is given by

$$
\begin{aligned}
v_{t, s} & :=\left(1, \ldots, r_{1}\right) \otimes\left(1, \ldots, s, r_{1}+1, \ldots, p, \bar{p}, \ldots, \overline{t+1}\right), \\
p & =\left(r_{1}+r_{2}+t-s\right) / 2
\end{aligned}
$$

Note that $p$ is a positive integer. Using these highest weight elements, we can show the following

Lemma 3.5. The value of the local energy function on $B^{r_{1}, 1} \otimes B^{r_{2}, 1}$ ıs given by

$$
H\left(b_{1} \otimes b_{2}\right)=-\left(\min \left(r_{1}, r_{2}\right)-s\right), \quad \text { if } b_{1} \otimes b_{2} \in B\left(\Lambda_{t}+\Lambda_{s}\right) .
$$

where we have normalized $H$ to be zero on the component $B\left(\Lambda_{r_{1}}+\Lambda_{r_{2}}\right)$.

Proof. Let $H_{t, s}$ be the value of $H$ on the $U_{q}\left(C_{n}\right)$ crystal $B\left(\Lambda_{t}+\Lambda_{s}\right)$. We shall show

(i) $H_{t+1, s+1}=H_{t, s}+1$,

$$
0 \leq s \leq t \leq n-1
$$

(ii) $H_{t-1, s+1}=H_{t, s}+1$,

$$
0 \leq s \leq t-2 \leq n-2 \text {. }
$$

from which the result will follow with the normalization $H_{r_{1}, r_{2}}=0$ (resp. $\left.H_{r_{2}, r_{1}}=0\right)$ if $r_{1} \geq r_{2}$ (resp. $r_{2} \geq r_{1}$ ).

To show (i), note that if

$$
\begin{aligned}
w_{1} & :=\left(\tilde{f}_{1} \cdots \tilde{f}_{s}\right)\left(\tilde{f}_{1} \cdots \tilde{f}_{s}\right)\left(\tilde{f}_{s+1} \cdots \tilde{f}_{t}\right) v_{t, s} \\
& =\left(1, \ldots, r_{1}\right) \otimes\left(2, \ldots, s+1, r_{1}+1, \ldots, p, \bar{p}, \ldots, \overline{t+2}, \overline{1}\right)
\end{aligned}
$$


then $\tilde{f}_{0} w_{1}=v_{t+1, s+1}$. Also we have

$$
w_{1} \mapsto\left(1, \ldots, r_{2}\right) \otimes\left(2, \ldots, s+1, r_{2}+1, \ldots, p, \bar{p}, \ldots, \overline{t+2}, \overline{1}\right)
$$

under the isomorphism $B^{r_{1}, 1} \otimes B^{r_{2} .1} \simeq B^{r_{2} .1} \otimes B^{r_{1}, 1}$, since both vectors are mapped to the 2 -column $C_{n}$ tableau with column reading $(2, \cdots, s+1,2, \cdots, t+$ 1) under the tableaux product (2.6) using $C_{n}$ insertion [Bak2, Lec]. The result now follows from the definition (3.1).

To show (ii), we first prove it in the special case when $t+s=r_{1}+r_{2}$. There, defining

$$
w_{2}:=\left(2, \ldots, r_{1}+1\right) \otimes\left(2, \ldots, s, r_{1}+2, \ldots, r_{1}+r_{2}-s+1, \overline{1}\right)
$$

we have

$$
\begin{aligned}
& v_{r_{1}+r_{2}-s+1 . s-1} \\
& =\left(\tilde{e}_{r_{1}+r_{2}-s+1} \cdots \tilde{e}_{r_{1}+1}\right)\left(\tilde{e}_{r_{1}} \cdots \tilde{e}_{1}\right)\left(\tilde{e}_{s-1} \cdots \tilde{e}_{1}\right)\left(\tilde{e}_{r_{1}+r_{2}-s+2} \cdots \tilde{e}_{n}\right)\left(\tilde{e}_{n-1} \cdots \tilde{e}_{1}\right) w_{2}
\end{aligned}
$$

However, we also have

$$
v_{r_{1}+r_{2}-s, 9}=\left(\tilde{e}_{r_{1}+r_{2}-s} \cdots \tilde{e}_{r_{1}+1}\right)\left(\tilde{e}_{r_{1}} \cdots \tilde{e}_{1}\right) \tilde{f}_{0} w_{2}
$$

Noting that

$$
w_{2} \mapsto\left(2, \ldots, r_{2}+1\right) \otimes\left(2, \ldots, s, r_{2}+2, \ldots, r_{1}+r_{2}-s+1, \overline{1}\right)
$$

again, since both vectors are mapped to the same 2-column tableau under the $C_{n}$ tableaux product, the result now follows in this special case. For arbitrary $t, s$ we then use (i) to show that (assuming $r 1 \geq r_{2}$ without loss of generality)

$$
\begin{aligned}
H_{t-1, s+1} & =H_{\left(r_{1}+r_{2}+t-s\right) / 2-1 .\left(r_{1}+r_{2}+s-t\right) / 2+1}-\left(r_{1}+r_{2}-t-s\right) / 2 \\
& =H_{\left(r_{1}+r_{2}+t-s\right) / 2 .\left(r_{1}+r_{2}+s-t\right) / 2}-\left(r_{1}+r_{2}-t-s\right) / 2+1=H_{t, s}+1
\end{aligned}
$$

Let us now show that in the case $r_{1}=r_{2}=n$, the value of $H$ can also be computed using the embedding $\psi$ of Prop. 2.2. First, some preparatory results are needed.

Proposition 3.6. Suppose $b_{\iota} \in B_{C}\left(\Lambda_{k_{1}}\right)$, for some $k_{l}, i=1, \ldots, 4$. Then

$$
b_{1} *_{C} b_{2}=b_{3} *_{C} b_{4} \quad \Longrightarrow \quad \psi\left(b_{1}\right) *_{A} \psi\left(b_{2}\right)=\psi\left(b_{3}\right) *_{A} \psi\left(b_{4}\right)
$$


Proof. We can use an inductive proof similar to that used in the proof of Prop. 2.3 (i') to reduce this to the case when $b_{1} \otimes b_{2}$ and $b_{3} \otimes b_{4}$ are highest weight elements. Such a case can then be proved by explicit calculation using the vectors (3.11).

Corollary 3.7. $\psi\left(b_{1} *_{C} b_{2}\right)=\psi\left(b_{1}\right) *_{A} \psi\left(b_{2}\right)$

Proof. Suppose $b_{1} *_{C} b_{2}=b_{4} \mid b_{3}$ as a two-column $C_{n}$ tableau. Then $b_{1} *_{C} b_{2}$ $=b_{3} *_{C} b_{4}$ and so by definition $\psi\left(b_{1} *_{C} b_{2}\right)=\psi\left(b_{3}\right) *_{A} \psi\left(b_{4}\right)=\psi\left(b_{1}\right) *_{A} \psi\left(b_{2}\right)$

For an element $b$ with column reading $P$, define

$$
\psi_{L}(b):=\frac{P_{+}}{\bar{Q}_{-}} \quad \psi_{R}(b):=\frac{\left(P_{-}\right)^{c}}{\left(\bar{Q}_{+}\right)^{c}}=\frac{Q_{+}}{\bar{P}_{-}}
$$

so that $\psi(b)=\psi_{L}(b) *_{A} \psi_{R}(b)$. We now have the main result in this section

Proposition 3.8. Let $p=b_{1} \otimes \cdots \otimes b_{M}$ be a path in the $U_{q}^{\prime}\left(C_{n}^{(1)}\right)$ crystal $\left(B^{n, 1}\right)^{\otimes M}$ and define the path

$$
T(p)=\psi_{L}\left(b_{1}\right) \otimes \psi_{R}\left(b_{1}\right) \otimes \cdots \otimes \psi_{L}\left(b_{M I}\right) \otimes \psi_{R}\left(b_{\Lambda I}\right)
$$

in the $U_{q}^{\prime}\left(A_{2 n-1}^{(1)}\right)$ crystal $\left(B^{n, 1}\right)^{\otimes 2 \Lambda I}$. Then $T(p)$ is classically admissible and

$$
\operatorname{ind}_{p}(i)=\operatorname{ind}_{T(p)}(2 i-1)=\operatorname{ind}_{T(p)}(2 i) \quad 1 \leq i \leq M
$$

and hence $E(T(p))=2 E(p)$.

Proof. First note the fact that $T(p)$ is classically admissible follows from Prop. 2.3 (ii). Next, let us first show that for any $b_{\imath} \in B^{n, 1}, i=1,2$ we have

$$
H_{C}\left(b_{1} \otimes b_{2}\right)=H_{A}\left(\psi_{R}\left(b_{1}\right) \otimes \psi_{L}\left(b_{2}\right)\right)
$$

When $r_{1}=r_{2}=n$, then in the decomposition (3.10) we have $s=t$. Suppose $b_{1} *_{C} b_{2} \in B\left(2 \Lambda_{s}\right)$ for some $s$, so that by Lemma 3.5 we have $H_{C}\left(b_{1} \otimes b_{2}\right)=-(n-$ $s)$. Using Corollary 3.7 we can construct $\psi\left(b_{1} *_{C} b_{2}\right)=\psi\left(b_{1}\right) *_{\dot{A}} \psi_{L}\left(b_{2}\right) *_{A} \psi_{R}\left(b_{2}\right)$ by column inserting $\psi_{L}\left(b_{2}\right)$, and then $\psi_{R}\left(b_{2}\right)$ into $\psi\left(b_{1}\right)$. Suppose $\psi_{R}\left(b_{1}\right) *_{A}$ $\psi_{L}\left(b_{2}\right) \in B_{A}\left(\Lambda_{v}+\Lambda_{2 n-v}\right)$ for some $0 \leq v \leq n$. From [Shi, SW] we have $H_{A}\left(\psi_{R}\left(b_{1}\right) \otimes \psi_{L}\left(b_{2}\right)\right)=-(n-v)$. We must show $v=s$. It is clear that $\psi^{\prime}\left(b_{1}\right) *_{A} \psi_{L}\left(b_{2}\right) \in B\left(\Lambda_{v}+\Lambda_{n}+\Lambda_{2 n-v}\right)$. If we now insert $\psi_{R}\left(b_{2}\right)$ into this tableau, the resulting tableau will have shape $\Lambda_{k_{1}}+\Lambda_{v+k_{2}}+\Lambda_{n+k_{3}}+\Lambda_{2 n-v+k_{4}}$. 
From the Column Bumping Lemma [Ful], and the fact that $\psi\left(b_{1} *_{C} b_{2}\right) \in$ $B_{A}\left(2 \Lambda_{s}+2 \Lambda_{2 n-s}\right)$, we must have $k_{1}=v, k_{3}=n-v, k_{2}=k_{4}=0$ and $v=s$. Thus (3.12) holds.

To complete the proof, note that

$$
H_{A}\left(\psi_{L}\left(b_{\imath}\right) \otimes \psi_{R}\left(b_{\imath}\right)\right)=0 \quad \forall i, 1 \leq i \leq M
$$

since $\psi_{L}\left(b_{\imath}\right) *_{A} \psi_{R}\left(b_{\imath}\right)=\psi_{R}\left(b_{\imath}\right) \mid \psi_{L}\left(b_{\imath}\right)$ as a 2 -column $U_{q}\left(A_{2 n-1}\right)$ tableau. Hence the result follows.

Now, having defined the classically admissible $U_{q}^{\prime}\left(A_{2 n-1}^{(1)}\right)$ path $T(p)$ we can construct a semi-standard tableau (also denoted $T(p)$ ) in the usual manner $[\mathrm{NY}]$, and the above Proposition tells us that charge $(T(p))=2 E(p)$.

\section{Acknowledgments}

The author thanks Y. Koga and M. Okado for providing access to some of their unpublished notes. He also thanks M. Okado for very helpful discussions. The financial support of the Japan Society for the Promotion of Science is acknowledged.

\section{Appendix $\mathbb{A}$}

In this Appendix, we give a proof of Prop. 3.3. Of course, the statements (i) and (ii) are well-known and have been proved (in a much more general situation) in [NY, HKKOTY]. However, we include proofs since they will be used to prove (iii) (which is only true when $b_{1}, b_{2}$ belong to the crystal of the spinor representations of $U_{q}\left(B_{n}\right)$ or $\left.U_{q}\left(D_{n}\right)\right)$.

Proof of Proposition 3.3(i). Referring to Fig. A.1, let $A$ be the lowest particle which is the beginning of a down line in $b_{1}$, and $B$ be the highest particle which is the end of a down in $b_{2}$. Then [NY, Lemma 5.11] $A$ lies above $B$. Let $C$ be the first particle above $A$ which is the beginning of an up $\mathrm{H}$-line, ending in $D$. If we draw the LS-line for such a particle, it must coincide with the H-line, since there are no particles in $b_{2}$ between $A$ and $D$ (if there were, there would be a up $\mathrm{H}$-line occurring between $A$ and $C$, contradicting the fact that $C$ is lowest). Thus we let $J(1)$ be the order of $C$. We continue in this manner with the next occurring up H-line above $C$, letting it be $J(2)$ and so on. All LS-lines constructed in this manner coincide with the H-lines. Such a process exhausts all the particles above $A$ in $b_{2}$, since there are no down $\mathrm{H}$-lines emanating from particles in $b_{2}$ above $A$ (since $A$ is above $B$ ). Next, we 

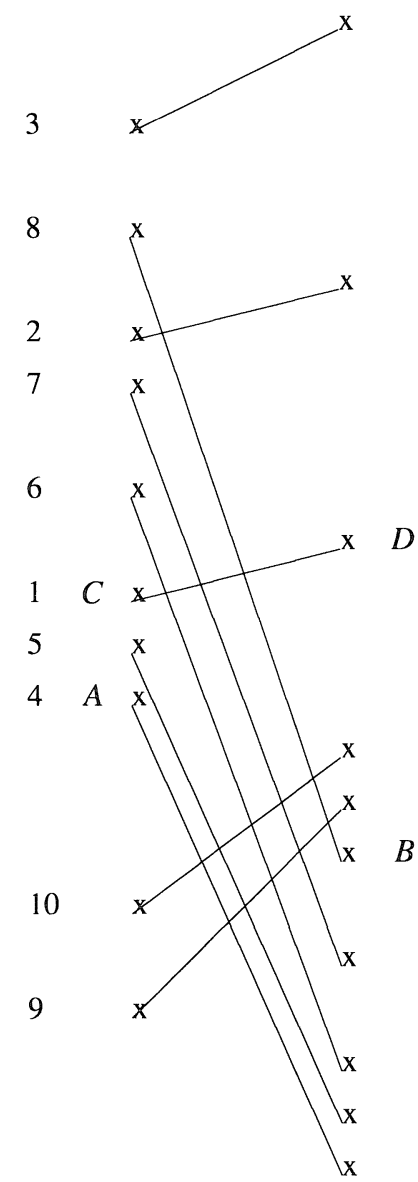

Figure A.1. Correspondence between $L S$ and $H$-diagrams

continue ordering the particles in $b_{1}$ by letting the next particle in the order $J$ be $A$ itself. Such an LS line from $A$ must connect to the lowest particle in $b_{2}$, since all particles above $A$ in $b_{2}$ are already occupied by LS lines, and the down LS line from $A$ is the first one. We then continue to draw down LS-lines for the remaining particles above $A$ in $b_{1}$ - these must coincide with the down $\mathrm{H}$-lines since none of the down $\mathrm{H}$-lines cross (since the H-diagram was drawn with top-to-bottom order). Finally, we continue the order $J$ from the lowest particle in $b_{1}$, continuing upward towards $A$. These up LS-lines must connect with particles in $b_{2}$ located between $A$ and $B$, and hence must coincide with 
the H-lines.

Proof of Proposition 3.3(ii). Suppose that for a given $L S$-diagram, we have 2 different orders $J$ and $J^{\prime}$ which differ by a transposition i.e. $J^{\prime}(i)=$ $J(i+1), J^{\prime}(i+1)=J(i)$ and $J(k)=J^{\prime}(k)$ for all $k \neq i, i+1$. We must show that the number of down lines remains the same with respect to either order. There are 4 possible cases to consider, depending on whether the lines emanating from the particles labeled $J(i)$ and $J(i+1)$ are up lines or down lines. The possible configurations are shown in Figs. A.2-A.5. Let us give a detailed explanation for Fig. A.3.

In Fig. A.3, in subcases (i), (ii) and (iv) $J(i+1)$ is above $J(i)$, while in subcase (iii) it is below. The particle at the end of the line coming from $J(i+1)$ is either below the particle at $J(i)$ (corresponding to subcases (i) and (ii)), or above the level of $J(i)$ (corresponding to subcases (iv)) in which case it must also be located above the end of the line emanating from $J(i)$. Also the subcases (i) and (ii) correspond to whether the particle on the right connecting to $J(i)$ is above or below $J(i+1)$. These exhaust all possibilities and in all subcases it is clear that the number of down lines remains the same.

Proof of Proposition 3.3(iii). The statement follows from the fact that for two orders $J$ and $J^{\prime}=s_{\imath} J$, the number of such lines in the LS-diagram of $b_{1} \otimes b_{2}$ with order $J$ remains unchanged when going to order $J^{\prime}$. This in turn follows from an examination of the diagrams in Figs. A.2-A.5 and the following lemma.

$\mathbb{L e m m a} \mathbb{A} . \mathbb{1}$. For $b_{1}, b_{2} \in B\left(\Lambda_{n}\right)$ for $U_{q}\left(B_{n}\right)$ or $U_{q}\left(D_{n}\right)$, or $b_{1}, b_{2} \in$ $B\left(\Lambda_{n-1}\right)$ for $U_{q}\left(D_{n}\right)$, the LS-diagram of $b_{1} \otimes b_{2}$ does not contain any subdiagrams of the form appearing in Fig. A.6

Proof. First note that the LS-diagram of any order $J$ can be obtained from the diagram for some fixed order $J_{0}$ by a sequence of elementary transpositions, the results of which appear in Figs. A.2-A.5. By examination of these diagrams, one sees that the result will follow from an induction argument, once we can prove it for some fixed order $J_{0}$.

Let us fix the order $J_{0}$ to be the bottom-to-top order. As such, we only have to consider Figs A.6(a),(b) and (d). Let us first consider Fig. A.6 (b). In such a case, let the particle on the left be located at level $\bar{b}$, and the one 
on the right at level $\bar{a}$. We consider two subcases, according to whether the first particle on the right appearing below level $b$ is connected to a particle on the left or not (see Figs. A.7(i) and (ii)). In the first case, locate the lowest particle connected such that all particles above it are also connected (it must appear above $\bar{a}$ ), and suppose such particle is at level $\bar{c}$. Suppose further, it is connected to a particle on the left at level $\bar{d}$. Clearly there are no particles (connected or unconnected) on the right between $\bar{d}$ and $\overline{c-1}$ (since, if there were any such particles, choose the highest - if it is connected, it would contradict the assumption that the connected particle at $\bar{c}$ was lowest; if it is unconnected, the particle on the left at $\bar{d}$ could not connect with the particle at $\bar{c}$ ). By the assumption all particles on the right appearing above $\bar{b}$ (including particles at unbarred levels) are connected to particles on the left between $\bar{c}$ and $\bar{b}$. In particular, the particles on the right between $d$ and $b$, and those between $\bar{b}$ and $\bar{d}$ are all connected and there are $b+1-d$ such particles (due to the fact that the columns represent "spinor" tableaux). However, these particles must connect with particles on the left which can only lie in the set $\{\bar{d}, \ldots, \overline{b-1}\}$, of cardinality at most $b-d$, whence a contradiction.

In the second subcase, suppose the highest particle on the right below $\bar{b}$ is unconnected and located at level $\bar{c}$ (certainly $\bar{c} \neq \bar{b}$, otherwise the particle on the left at $\bar{b}$ would connect horizontally, and not downwards as assumed). Again, from the spinor condition, there are particles on the right at all levels $c+1, \ldots, b$ and they must connect with particles between $\overline{c+1}$ and $\overline{b-1}$, and so by cardinality considerations, there is a contradiction. A similar argument with two subcases can be used to show that the configuration in Fig. A.6 (a) cannot appear.

Let us conclude by examining Fig. A.6 (d). Using the bottom-to-top order, there are two possibilities for the particles with order $J(i)$ and $J(i+1)$, namely the particle of order $J(i)$ is at some level $\bar{a}(1 \leq a<n)$ and that of order $J(i+1)$ is at level $n$, or $J(i)$ is at level $\bar{n}$, and $J(i+1)$ is at level $a(1 \leq a<n)$. We consider the first case only, as the proof of the second is similar.

Let the up-line from $\bar{a}$ end at a particle at level $c$, and the down-line from the particle at $n$, end at a particle at level $\bar{b}$. As in the paragraph above, we subdivide into two subcases, in accordance with whether the first particle on the right below $\bar{a}$ is connected or not. If it is connected, locate the lowest connected particle below $\bar{a}$ such that all particles above it are also connected. Suppose this particle appears at level $\bar{e}$ and is comnected to a particle at level $\bar{d}$ on the left. We further subdivide into 3 cases according to whether (i) $c \leq d<a$ (ii) $d<c \leq a$ or (iii) $d<a \leq c$. For all cases, we note that there must be no 
particles on the right at levels $1, \ldots, c-1$, hence all levels $\overline{1}, \ldots, \overline{c-1}$ on the right have a particle. Also, in all cases we must have $\bar{b}$ below $\bar{d}$.

Beginning with case (i) (refer to Fig A.8(i)), we see that the set of particles on the right between $d$ and $a$ and between $\bar{a}$ and $\bar{d}$ are all connected and such a set has cardinality $a+1-d$. But such a set must connect with particles on the left lying between the levels $\bar{d}$ and $\overline{a-1}$, which is a contradiction.

In case (ii) (see Fig A.8(ii)), since all the levels below $\bar{c}$ on the right have a particle, the particles between $\bar{d}$ and $\overline{c-1}$ are connected by horizontal lines. As such, all particles between $c$ and $n$, and between $\bar{n}$ and $\bar{c}$ on the right are connected to particles on the left between $\bar{c}$ and $\overline{a-1}$. Since we know $a \leq n-1$, we have another cardinality contradiction.

In case (iii), all levels on the right between $\overline{a-1}$ and $\bar{d}$ have particles, which must be connected by horizontal lines to particles on the left at the same levels. However, there must also be a particle on the right at level $\bar{a}$, and this must be connected on the particle on the left at level $\bar{a}$, contradicting the fact that the latter particle is connected to a particle at $c$ on the right.

As mentioned above, we must also consider the case when the first particle on the right below $\bar{a}$ is unconnected, in which case we have another 3 subcases, but the details are similar and are hence omitted.

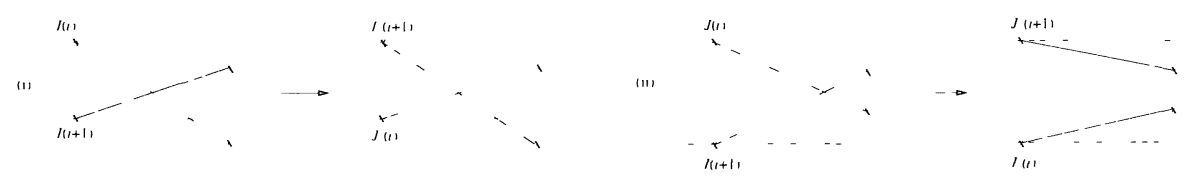

Figure A.2. $J(i)$ down, $J(i+1)$ up

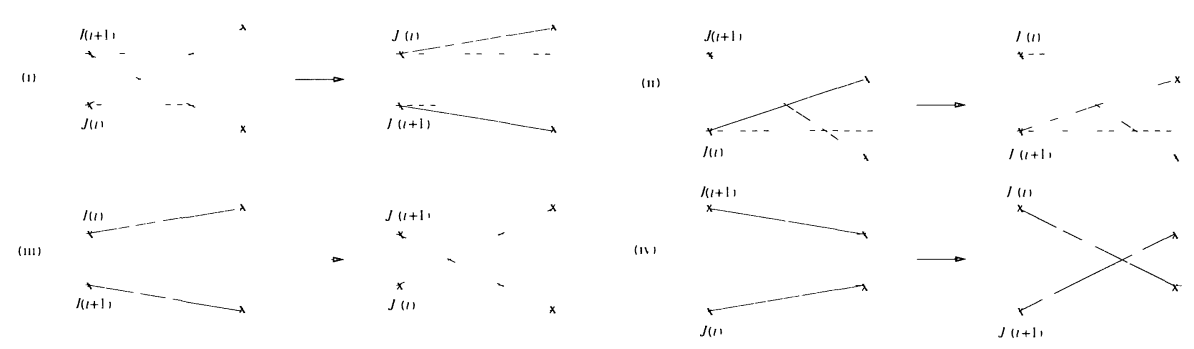

Figure A.3. $J(i)$ up, $J(i+1)$ down 


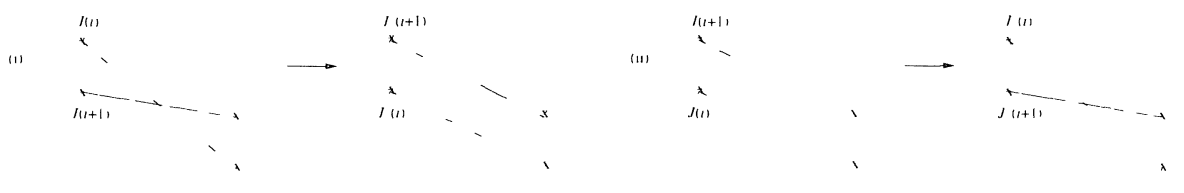

Figure A.4. $J(i)$ down, $J(i+1)$ down

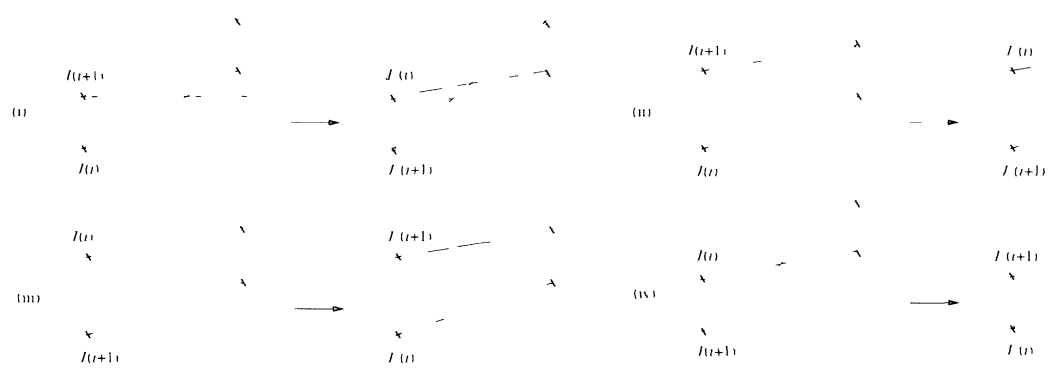

Figure A.5. $J(i)$ up, $J(i+1)$ up

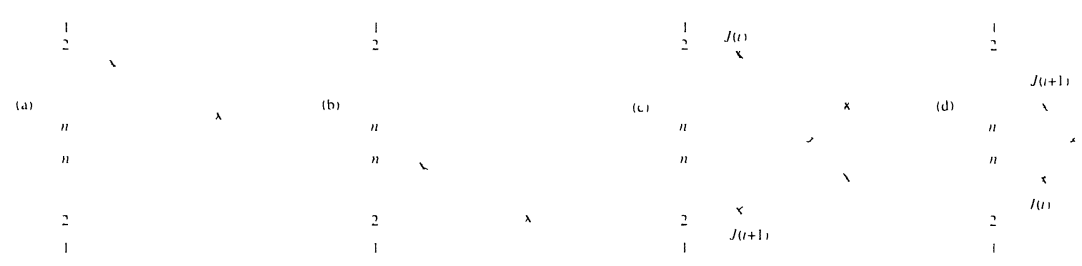

Figure A.6. Configurations not appearing in LS diagrams for spinor reps. 
(1)
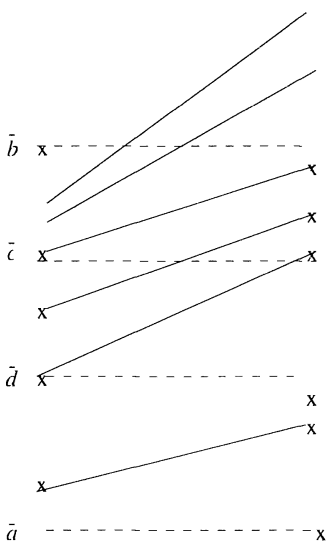

(11)
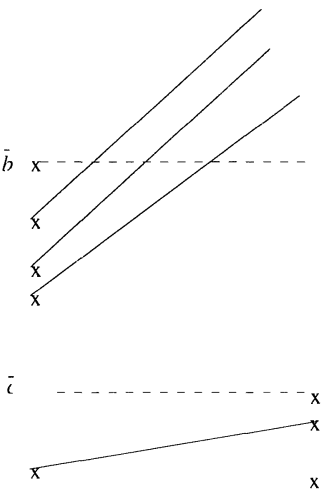

$\bar{a} \ldots \ldots \ldots-\ldots$

Figure A.7.
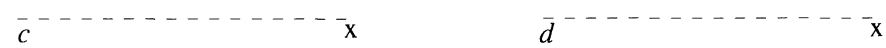

(i)

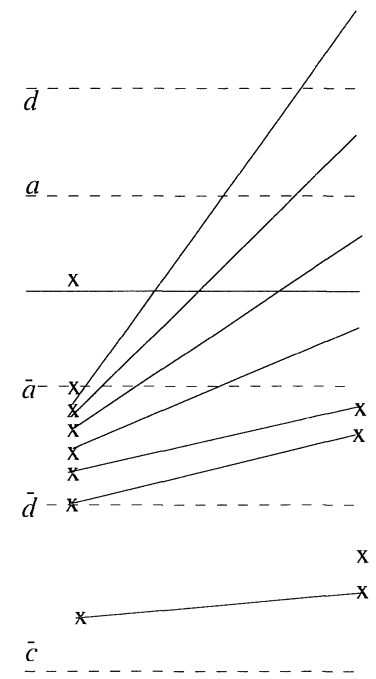

(ii)
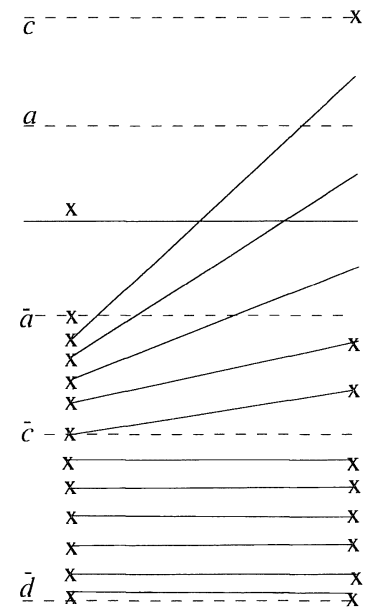

Figure A.8. 


\section{References}

[Bakl] Baker, T. H., Combinatorics of crystals for tensor and spinor representations of $U_{q}\left(B_{n}\right)$, to appear in Proc. of the International Workshop on Specıal Functions, Hong Kong, 1999

[Bak2] -, An insertion scheme for $C_{n}$ crystals, RIMS preprint 1234 (1999).

[Con] Concini, C. De, Symplectic standard tableaux, Adv. un Math., 34 (1979), 1-27.

[DS] Dulucq, S. and Sagan, B. E., La correspondance de Robinson-Schensted pour les tableaux oscillants gauches, Discr. Math., 139 (1995), 129-142.

[FOY] Fukuda, K., Okado, M. and Yamada, Y., Energy functions in box ball systems, Math.QA/9908116.

[FSS] Frappat, L., Sorba, P. and Sciarrino, A., A crystal base for the genetic code, Phys. Lett. A, 250 (1999), 214-221.

[Ful] Fulton, W., Young Tableaux, Cambridge University Press, 1st edition, 1997.

[HKKOT] Hatayama, G., Koga, Y., Kuniba, A., Okado, M. and Takagi, T.. Finite crystals and paths, Math.QA/9901082.

[HKKOTY] Hatayama, G., Kirillov, A. N., Kuniba, A., Okado, M., Takagi, T. and Yamada, Y., Character formulae of $\hat{s l}_{n}$-modules and inhomogeneous paths, Nucl. Phys. B. 536 (1999), 575-616.

[HKOTY] Hatayama, G., Kuniba, A., Okado, M., Takagi, T. and Yamada, Y., Remarks on fermionic formula, Contemp. Math., 248 (1999), 243-291.

[HKT] Hatayama, G., Kuniba, A. and Takagi, T., Soliton cellular automata associated with finite crystals, Solv-int/9907020.

[Kas] Kashiwara, M., Similarity of crystal bases, Contemp. Math., 194 (1996), $177-$ 186.

[KMN1] Kang, S-J., Kashiwara, M., Misra, K., Miwa, T., Nakashima, T. and Nakayashiki, A., Affine crystals and vertex models, Int. J. Mod. Phys. A, 7, Suppl. 1 A (1992), 449-484.

[KMN2] Kang, S-J., Kashiwara, M., Misra, K., Miwa, T., Nakashima, T. and Nakayashiki, A., Perfect crystals of quantum affine Lie algebras, Duke Math. J., 68 (1992), 499-607.

[KN] Kashiwara, M. and Nakashima, T., Crystal graphs for representations of the $q$-analogue of classical Lie algebras, J. Alg., 165 (1994), 295-345.

[Lec] Lecouvey, C., Schensted-type correspondence, plactic monoid and jeu-de-taquin for type $C_{n}$, Uni. Caen preprint, 1999.

[LS] Lascoux, A. and Schützenberger, M. P., Sur une conjecture de H. O. Foulkes, C. R. Acad. Scı. Parıs, 286A (1978), 323-324.

[Mac] Macdonald, I. G., Symmetrıc functıons and Hall polynomıals, Oxford University Press, Oxford, 2nd edition, 1995.

[Nak] Nakashima, T., Crystal base and a generalization of Littlewood-Richardson rule for the classical Lie algebras, Commun. Math. Phys., 154 (1993), 215-243.

[NY] Nakayashiki, A. and Yamada, Y., Kostka polynomials and energy functions in solvable lattice models, Selecta Math., New Ser., 3 (1997), 547-599.

[Oka] Okado, M., Quantum $R$ matrices associated to spin representations of $B_{n}$ and $D_{n}$, Comm. Math. Phys., 134 (1990), 467-486.

[Rob] Roby, T., The connection between the Robinson-Schensted correspondence for skew oscillating tableaux and graded graphs, Discr. Math., 139 (1995), 481-485.

[She] Sheats, J. T., A symplectic jeu-de-taquin bijection between the tableaux of King and of De-Concini, Trans. Amer. Math. Soc., 351 (1999), 3569-3607.

[Shi] Shimozono, M., Affine type A crystal structure on tensor products of rectangles, Demazure characters, and nilpotent varieties. Math.QA/9804039, 1998.

[Sun] Sundaram, S., The Cauchy identity for $s p(2 n)$, J. Comb. Th. A, $\mathbf{5 3}$ (1990), 209-238.

[SW] Schilling, A. and Warnaar, S. O., Inhomogeneous lattice paths, generalized Kostka polynomials and $A_{n-1}$ supernomials, Comm. Math. Phys., 202 (1999), 
359-401. 